\title{
Electroosmotic flow of a two-layer fluid in a slit channel with gradually varying wall shape and zeta potential
}

\author{
Cheng Qi ${ }^{a 1}$, Chiu-On $\mathrm{Ng}^{b}$ \\ ${ }^{a}$ Hubei Key Laboratory of Theory and Application of Advanced Materials Mechanics, \\ Wuhan University of Technology, Wuhan 430070, China \\ ${ }^{b}$ Department of Mechanical Engineering, The University of Hong Kong, \\ Pokfulam Road, Hong Kong
}

\begin{abstract}
This study aims to investigate electroosmotic (EO) flow of a two-layer fluid through a slit microchannel where the wall shape as well as zeta potential may vary slowly and periodically with axial position. The two-layer EO flow is a model for the flow of two immiscible fluids: a non-conducting working fluid being dragged into motion by a conducting sheath fluid. Electric double layers may develop in the conducting fluid near the interface between the two fluids, and near a wall that is assumed to be nonuniform in both shape and zeta potential distribution. Because of these geometrical and electrical non-uniformities, pressure is internally induced. The two-fluid flow is therefore driven by electrokinetic and hydrodynamic forcings, while subjected to the combined effects of the axial variations of the wall shape and potential distribution. The present problem is formulated by invoking the lubrication approximation, for a nearly parallel flow of low Reynolds number, and the governing equations are solved as analytically as possible. The induced pressure gradient and the deformed shape of the interface, which are functions of axial position, as well as the flow rates of the two fluids, are determined by an iterative trial-and-error numerical scheme. Results are generated to show the effects due to various factors, including the applied pressure difference, interfacial potential, viscosity ratio, wall undulation, and phase shift between the wall shape and potential distribution. Some of the effects on flow in a non-uniform channel can be qualitatively different from that in a uniform channel.
\end{abstract}

Keywords: electroosmotic flow; electric double layer; two-fluid flow; wall patterns.

\footnotetext{
${ }^{1}$ Corresponding author. E-mail address: qicheng8912@163.com (C. Qi).
} 


\section{Introduction}

Electrokinetic pumping has now been widely used in the transport of fluid in microfluidic devices. Under an applied electric field, fluid is driven into motion through Lorentz force acting on unbalanced free ions in an electric double layer (EDL) that develops in the vicinity of a solid-liquid interface. The resulting flow, known as electroosmotic (EO) flow, offers some outstanding features. First, the velocity profile in an EO flow is nearly plug-like, ensuring a low sample dispersion [1] and avoiding mixing and rheological changes produced by lateral velocity variations [2]. Second, the section-mean velocity is almost independent of the channel size, which is desirable for fluid transport in an ultrafine channel. Other advantages include a more precise control of flow magnitude and direction, few mechanical moving parts (thus less mechanical failures), easy fabrication, high reliability, little noise, and so on. These advantages have made EO a promising pumping mechanism for a wide range of applications, ranging from drug delivery [3] to chip cooling [4].

EO pumping can be applied not only to single fluids, but also to a two-layer fluid $[5,6]$. Some non-polar fluids with very low ionic conductivity, such as oil, ethanol and organic solvents, do not develop EDLs [7], and hence cannot be pumped by EO forcing directly. Nevertheless, EO effect can serve as an indirect actuation mechanism to pull non-polar fluids into motion via some sheath liquid with significant ionic conductivity. This idea was practically realized by Brask et al. [8] and Watanabe et al [9]. Pioneering theoretical studies were conducted by Gao et al. [5] and Ngoma and Erchiqui [7], who looked into steady two-layer EO flows in a rectangular microchannel and between two parallel plates. These authors, however, ignored electrostatic contribution at the liquid-liquid interface. The effect of Maxwell stress, which is caused by the interaction between free charges on a liquid-liquid interface and the externally applied electric field, is found to be significant and has to be taken into consideration [10,11]. This has motivated Gao et al. [12] to incorporate the Maxwell stress into the interfacial condition in their study on transient two-layer EO flow. Li et al. [13,14] further proposed a three-layer-fluid model for EO flow of a layer of non-conducting fluid bounded by two layers of conducting fluid in a rectangular microchannel. Liu et al. [15] developed analytical models for two-layer EO flow in a circular microchannel, where Newtonian-Newtonian and Newtonian-Casson fluids were examined. The studies by Choi et al. [16] and Gaikwad 
et al. [17] were focused on the zeta-potential jump and the interfacial hydrodynamic slip on the liquid-liquid interface for two-layer EO flow in a parallel-plate channel.

The above-mentioned theoretical studies have only considered uniform zeta potential at flat walls, or a plane interface between two immiscible fluids. Mandal et al. [18] analyzed two-layer EO flow between two parallel plates in the presence of axially varying zeta potentials. They obtained analytical solutions for small deformation of the interface through an asymptotic analysis, and applied the phase field formalism to the case of large interfacial deformation. In addition to surface charge modulation, which itself can offer a wealth of interesting flow patterns, the problem will become more interesting when wall undulation is considered as well. Ajdari $[19,20]$ found that the combined effect of these two wall patterns can generate a net flow even when the walls are on average electro-neutral. Axially modulated zeta potentials with a zero average alone can only give rise to recirculation rolls, but not a net flow since the flux/reflux caused by equal positive/negative surface charges will exactly balance each other. A net flow may happen only when this symmetry is broken by the superposition of undulated walls. Chen and Cho [21] further investigated steady-state mixing characteristics for EO flow with heterogeneous charge patterns on wavy surfaces. Their numerical results suggest that the mixing performance can be remarkably improved by increasing either the wave amplitude or the length of the wavy surface or the magnitude of the heterogeneous zeta potential. Other authors who also looked into the combined effect of wall shape and charge patterns on EO flow include, among others, Xuan and Li [22], Bhattacharyya and Nayak [23], Ng and Qi [24], Bhattacharyya and Bera [25], and Yoshida et al [26]. All these studies, however, only considered single-fluid EO flow.

Motivated by previous studies on the interaction between wall undulation and charge modulation, this paper aims to look into such interaction in a two-fluid configuration of EO flow. In order to enable the present problem to be solved as analytically as possible, we shall adopt the lubrication approximation $[27,28]$ to simplify the problem. The lubrication technique has been utilized previously by various authors to deal with EO flow in non-uniform channels; examples include Long et al. [29] and Ajdari [30]. For EO flow in microchannels with arbitrary cross-sectional geometry and arbitrary surface charge distribution, Ghosal [31] obtained asymptotic solutions with the aid of the lubrication approximation. Essentially this 
approximation is to reduce two-dimensional flow to quasi-one-dimensional flow. Its validity is based on two conditions. One condition is a sharp contrast in length scales for much slower variations in the axial than the transverse directions. Another condition requires the Reynolds number to be sufficiently small for negligible inertia.

For EO flow of two immiscible fluids in a uniform channel, the liquid-liquid interface is a flat surface [5-7,12-17]. If axial variations in wall shape and/or wall charge are allowed, the interface between the two immiscible fluids will change in shape as a function of axial position. The deformed shape of the interface is part of the flow structure, and hence has to be found as part of the solution. In the present study, the lower wall of a slit channel is assumed to be patterned with periodic variations in both wall shape and wall potential, and these two periodic functions, one geometrical and one electrical, have the same wavelength but with a possible phase shift between them. The displacement of the interface, under the combined effects due to wall undulation and charge modulation, may give rise to a variety of interesting features. As shown by Mandal et al. [18], a strongly deformed interface is usually associated with a complex flow structure, where the deformation can be affected by fluid properties, such as viscosities and permittivities of the two fluids. These authors also found that an increase in the wavelength of wall charge modulation may result in significant augmentation of the interface deformation. These previous findings have guided us to define the scope of the present problem, which is formulated such that it can be solved as analytically as possible, and yet it can reveal the effects of various geometrical, electrical, and hydrodynamic factors on a two-layer EO flow.

Our problem is described in further detail in Sec. 2. On the basis of the lubrication approximation (i.e., a nearly parallel flow with negligible inertia), the Poisson-Boltzmann and momentum equations are formally solved for the potential distribution and axial velocity for the two fluids. The special case for two-layer EO flow through a uniform channel is presented in Sec. 3. An iterative trial-and-error numerical scheme, which is used to determine the pressure gradient and interface shape as functions of axial position, is then introduced in Sec. 4. Numerical results are presented in Sec. 5, where we examine how different parameters (including the ratio of viscosities, applied pressure drop, interfacial potential, and parameters pertaining to the wall patterns) may have various effects on the two-fluid EO flow. 


\section{Problem formulation}

Our problem is to investigate EO flow of a two-layer fluid in a non-uniform slit microchannel, where the two fluids are of distinct chemical and physical properties and separated by a sharp interface. The channel is non-uniform in both height and wall potential distribution, which may vary slowly and periodically with axial position. Figure 1 depicts a definition sketch of our problem, where the axial $x$-axis is positioned along the upper planar wall, and the transverse $y$-axis points vertically downward. The lower wall has a wavy shape given by $y=w(x)$, and a wall potential given by $\zeta_{2}=\zeta_{2}(x)$. These two functions are periodic functions of $x$ with the same wavelength, denoted by $L$. The amplitude of the wavy wall is $w_{0}$ (or the bottom groove depth is $2 w_{0}$ ). The zeta potential at the liquid-liquid interface is a known constant, denoted by $\zeta_{1}$. The flow is driven under the combined action of a pressure gradient, $\Delta P / L$, and an electric field, $E_{x}$, which are applied externally along the $x$-direction. It is also assumed that the lower fluid is conducting while the upper fluid is non-conducting. In other words, the electric Lorentz force is non-zero in the former, but is zero in the latter. In a static state, the interface between the fluids is a flat surface located at $y=h_{w}$. When the fluids are in motion, the interface between them will deform to a curved surface, $y=h(x)$, which is not known a priori and has to be found as part of the solution. The local thickness of the upper layer is therefore $h(x)$, while that of the lower layer is $w(x)-h(x)$. The height of the mean position of the interface above the crest of the bottom topography is denoted by $h_{0}$. In this problem, conditions pertaining to the lubrication approximation are assumed. It is assumed that the length scale for velocity variations in the axial direction is much longer than that in the transverse direction, $L \gg h_{w}$, which essentially implies that the flow is nearly one-dimensional: axial velocity is in general much larger in magnitude than transverse velocity. It is also assumed that the Reynolds number is so small that the inertia can be ignored. For a mildly curved interface, surface tension can also be ignored.

\section{$2.1 \quad$ Electric potential}

An electric double layer (EDL) forms in the conducting fluid in the vicinity of the liquidsolid and liquid-liquid interfaces. By electrostatics, the electric potential $\psi$ is related to the 


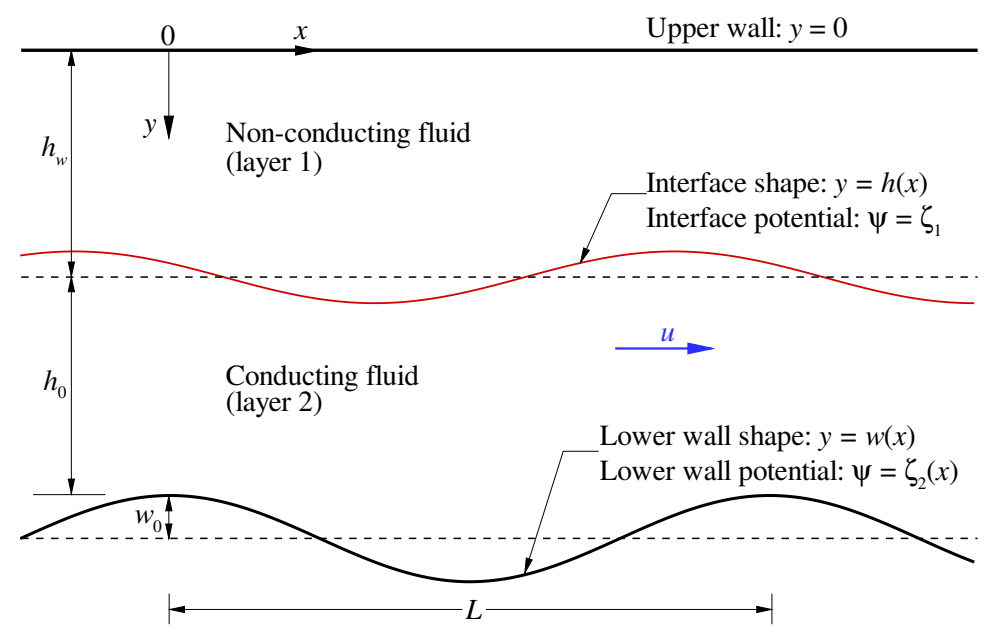

Figure 1: Definition sketch of the present problem: two-fluid electroosmotic flow in a slit channel with gradually varying shape and potential on the lower wall, where the upper fluid is non-conducting and the lower fluid is conducting. The upper dashed line denotes the mean position of the interface between the two fluids, and the lower dashed line denotes the mean position of the lower wall.

net charge density, $\rho_{e}$, by the Poisson equation as follows (after applying the lubrication approximation):

$$
\frac{\partial^{2} \psi}{\partial y^{2}}=-\frac{\rho_{e}}{\epsilon}
$$

where $\epsilon$ is the dielectric permittivity of the conducting fluid. For a binary electrolyte of $z: z$ symmetric valence, the free charge density is given by, under the condition of a Boltzmann distribution for ions in the EDL,

$$
\rho_{e}=-2 z e n_{\infty} \sinh \left(\frac{z e \psi}{k_{B} T}\right)
$$

where $e$ is the fundamental charge, $z$ is the valence of the fluid, $n_{\infty}$ is the bulk ionic concentration, $k_{B}$ is the Boltzmann constant and $T$ is the absolute temperature. Substituting Eq. (2) into Eq. (1) will form the well-known Poisson-Boltzmann equation. For sufficiently low wall potentials, as is assumed in the present study, the Poisson-Boltzmann equation can be linearized as follows, known as the Debye-Hückel approximation,

$$
\frac{\partial^{2} \psi}{\partial y^{2}}=\kappa^{2} \psi
$$


in which $\kappa=\left(2 n_{\infty} z^{2} e^{2} / \epsilon k_{B} T\right)^{1 / 2}$ is termed the Debye parameter, the inverse of which is the Debye shielding length of the EDL. The equation above can be readily solved, giving the electric potential distribution as follows:

$$
\psi(x, y)=\zeta_{1} \frac{\sinh [\kappa(w-y)]}{\sinh [\kappa(w-h)]}+\zeta_{2} \frac{\sinh [\kappa(y-h)]}{\sinh [\kappa(w-h)]} \quad \text { for } h(x) \leq y \leq w(x),
$$

which satisfies the boundary conditions

$$
\begin{array}{ll}
\psi=\zeta_{2}(x) & \text { at } y=w(x), \\
\psi=\zeta_{1} & \text { at } y=h(x),
\end{array}
$$

in which $\zeta_{2}$ is the zeta potential on the lower wall and $\zeta_{1}$ is the interfacial zeta potential. The EDLs formed near the lower wall and the interface between the two immiscible fluids are assumed to be so thin that they do not overlap each other. Generally, the zeta potential at the solid-liquid interface depends on the material properties of the solid wall and the fluid ionic properties [32]. The zeta potential $\zeta_{2}$, as mentioned before, is assumed to be nonuniformly distributed along the lower wall, i.e., $\zeta_{2}=\zeta_{2}(x)$. Variations in the solid-liquid zeta potential can be realized practically by either coating the solid surface with different materials or applying appropriate surface-chemistry treatments, or both [33-36]. Also, the wavy shape of the lower wall can be fabricated by following the procedures as described by Duffy et al. [37]. The zeta potential between the two immiscible liquids, $\zeta_{1}$, depends on the ionic properties of the fluids, the $\mathrm{pH}$ value and the concentration of electrolyte [38]. In the present study, we assume that this interfacial zeta potential is a known constant.

The electric potential can be written in a dimensionless form as follows:

$$
\hat{\psi}(\hat{x}, \hat{y})=\hat{\zeta}_{1} \frac{\sinh [\hat{\kappa}(\hat{w}-\hat{y})]}{\sinh [\hat{\kappa}(\hat{w}-\hat{h})]}+\hat{\zeta}_{2} \frac{\sinh [\hat{\kappa}(\hat{y}-\hat{h})]}{\sinh [\hat{\kappa}(\hat{w}-\hat{h})]} \quad \text { for } \hat{h}(\hat{x}) \leq \hat{y} \leq \hat{w}(\hat{x})
$$

where

$$
\left(\hat{\psi}, \hat{\zeta}_{1}, \hat{\zeta}_{2}\right)=\left(\psi, \zeta_{1}, \zeta_{2}\right) / \zeta_{0}, \quad(\hat{y}, \hat{w}, \hat{h})=(y, w, h) / h_{w}, \quad \hat{x}=x / L, \quad \hat{\kappa}=\kappa h_{w}
$$

and $\zeta_{0}$ is a characteristic scale for the electric potential. 


\subsection{Fluid flow}

Under the conditions pertaining to the lubrication approximation, the axial momentum equation for steady flow of the two fluids reads as below:

$$
\begin{gathered}
0=-\frac{\partial p_{1}}{\partial x}+\mu_{1} \frac{\partial^{2} u_{1}}{\partial y^{2}} \quad \text { for } 0 \leq y \leq h(x), \\
0=-\frac{\partial p_{2}}{\partial x}+\mu_{2} \frac{\partial^{2} u_{2}}{\partial y^{2}}+\rho_{e} E_{x} \quad \text { for } h(x)<y \leq w(x),
\end{gathered}
$$

where $p$ is the pressure (including both the applied and induced pressure), $u$ is the axial velocity, $\mu$ is the dynamic viscosity, $E_{x}$ is the applied electric field, and subscripts "1" and " 2 " are used to denote quantities of the non-conducting and conducting fluids, respectively.

To facilitate analysis, the following dimensionless quantities (distinguished by an overhead caret) are introduced

$$
\left(\hat{u}_{1}, \hat{u}_{2}\right)=\left(u_{1}, u_{2}\right) / u_{0}, \quad\left(\hat{K}_{1}, \hat{K}_{2}\right)=\left(K_{1}, K_{2}\right) /\left(\mu_{2} u_{0} / h_{w}^{2}\right)
$$

where $K_{i}(x)=-\partial p_{i} / \partial x(i=1,2)$ are the pressure gradients, and $u_{0}=-\epsilon E_{x} \zeta_{0} / \mu_{2}$, known as the Helmholtz-Smoluchowski or EO slip velocity, is chosen as the characteristic velocity. In terms of dimensionless quantities, Eqs. (8) and (9) become

$$
\begin{gathered}
0=\hat{K}_{1}+\frac{1}{\eta} \frac{\partial^{2} \hat{u}_{1}}{\partial \hat{y}^{2}} \quad \text { for } 0 \leq \hat{y} \leq \hat{h}(\hat{x}), \\
0=\hat{K}_{2}+\frac{\partial^{2} \hat{u}_{2}}{\partial \hat{y}^{2}}+\frac{\partial^{2} \hat{\psi}}{\partial \hat{y}^{2}} \quad \text { for } \hat{h}(\hat{x})<\hat{y} \leq \hat{w}(\hat{x}),
\end{gathered}
$$

where

$$
\eta=\mu_{2} / \mu_{1}
$$

is a ratio of the two dynamic viscosities, and the electric forcing has been replaced by $\rho_{e} E_{x}=$ $-\epsilon E_{x}\left(\partial^{2} \psi / \partial y^{2}\right)$ according to the Poisson equation. The solution to Eqs. (11) and (12) can be readily found to be

$$
\begin{gathered}
\hat{u}_{1}=\eta\left(-\frac{1}{2} \hat{K}_{1} \hat{y}^{2}+C_{1} \hat{y}+C_{2}\right) \quad \text { for } 0 \leq \hat{y} \leq \hat{h}(\hat{x}), \\
\hat{u}_{2}=-\frac{1}{2} \hat{K}_{2} \hat{y}^{2}-\hat{\psi}+C_{3} \hat{y}+C_{4} \quad \text { for } \hat{h}<\hat{y}(\hat{x}) \leq \hat{w}(\hat{x}),
\end{gathered}
$$

where $C_{1,2,3,4}$ are undetermined coefficients, and $\hat{\psi}$ is the electric potential given by Eq. (6). 
On the upper and lower walls, the no-slip boundary condition requires that

$$
\begin{gathered}
\hat{u}_{1}=0 \quad \text { at } \hat{y}=0, \\
\hat{u}_{2}=0 \quad \text { at } \hat{y}=\hat{w}(\hat{x}) .
\end{gathered}
$$

On the interface between the two immiscible liquids, $\hat{y}=\hat{h}(\hat{x})$, the matching of axial velocity gives

$$
\hat{u}_{1}=\hat{u}_{2}
$$

and the balance of shear stress (see details in the appendix) gives

$$
\frac{\partial \hat{u}_{1}}{\partial \hat{y}}=\eta \frac{\partial \hat{u}_{2}}{\partial \hat{y}}+\eta \frac{\partial \hat{\psi}}{\partial \hat{y}}
$$

which incorporates contributions from the Maxwell as well as viscous stresses.

The conditions prescribed in Eqs. (16)-(19) enable us to express the coefficients $C_{1,2,3,4}$ as follows:

$$
\begin{gathered}
C_{1}=\frac{\hat{h}[2 \hat{w}-(2-\eta) \hat{h}] \hat{K}_{1}+(\hat{w}-\hat{h})^{2} \hat{K}_{2}-2 \hat{\zeta}_{1}+2 \hat{\zeta}_{2}}{2[\hat{w}-(1-\eta) \hat{h}]}, \\
C_{2}=0, \\
C_{3}=\frac{-\eta \hat{h}^{2} \hat{K}_{1}+\left[\hat{w}^{2}-(1-2 \eta) \hat{h}^{2}\right] \hat{K}_{2}-2 \hat{\zeta}_{1}+2 \hat{\zeta}_{2}}{2[\hat{w}-(1-\eta) \hat{h}]}, \\
C_{4}=\frac{\eta \hat{h}^{2} \hat{w} \hat{K}_{1}+\hat{h} \hat{w}[(1-2 \eta) \hat{h}-(1-\eta) \hat{w}] \hat{K}_{2}+2\left[\hat{w} \hat{\zeta}_{1}-(1-\eta) \hat{h} \hat{\zeta}_{2}\right]}{2[1-\eta) \hat{h}]} .
\end{gathered}
$$

Note that $C_{1,2,3,4}$ are functions of $\hat{x}$. Our next task is to formulate equations to solve for the position of the interface $\hat{h}(\hat{x})$.

The volumetric fluxes of the conducting and non-conducting fluids can be found by integrating the axial velocities across the respective layers:

$$
\begin{aligned}
\hat{q}_{1} & =\int_{0}^{\hat{h}} \hat{u}_{1} \mathrm{~d} \hat{y} \\
& =\frac{\eta \hat{h}^{2}\left\{\hat{h}[4 \hat{w}-(4-\eta) \hat{h}] \hat{K}_{1}+3(\hat{w}-\hat{h})^{2} \hat{K}_{2}\right\}}{12[\hat{w}-(1-\eta) \hat{h}]}-\frac{\eta \hat{h}^{2}\left(\hat{\zeta}_{1}-\hat{\zeta}_{2}\right)}{2[\hat{w}-(1-\eta) \hat{h}]},
\end{aligned}
$$




$$
\begin{aligned}
\hat{q}_{2}= & \int_{\hat{h}}^{\hat{w}} \hat{u}_{2} \mathrm{~d} \hat{y} \\
= & \frac{(\hat{w}-\hat{h})^{2}\left\{3 \eta \hat{h}^{2} \hat{K}_{1}+(\hat{w}-\hat{h})[\hat{w}-(1-4 \eta) \hat{h}] \hat{K}_{2}\right\}}{12[\hat{w}-(1-\eta) \hat{h}]} \\
& +\frac{(\hat{w}-\hat{h})\left\{(\hat{w}-\hat{h}) \hat{\zeta}_{1}+[\hat{w}-(1-2 \eta) \hat{h}] \hat{\zeta}_{2}\right\}}{2[\hat{w}-(1-\eta) \hat{h}]}-\frac{\hat{\zeta}_{1}-\hat{\zeta}_{2}}{\hat{\kappa}} \tanh \left[\frac{\hat{\kappa}}{2}(\hat{w}-\hat{h})\right] .
\end{aligned}
$$

Note that, by virtue of continuity, $\hat{q}_{1}$ and $\hat{q}_{2}$ are independent of $\hat{x}$. On rearranging the above two equations, the pressure gradients can be expressed as

$$
\begin{gathered}
\hat{K}_{1}(\hat{x})=\frac{3(\hat{w}-\hat{h})[\hat{w}-(1-4 \eta) \hat{h}] \hat{q}_{1}-9 \eta \hat{h}^{2} \hat{q}_{2}}{\eta \hat{h}^{3}(\hat{w}-\hat{h})[\hat{w}-(1-\eta) \hat{h}]}+\frac{3\left(2 \hat{\zeta}_{1}+\hat{\zeta}_{2}\right)}{\hat{h}[\hat{w}-(1-\eta) \hat{h}]} \\
\quad-\frac{9\left(\hat{\zeta}_{1}-\hat{\zeta}_{2}\right) \tanh \left[\frac{\hat{\kappa}}{2}(\hat{w}-\hat{h})\right]}{\hat{\kappa} \hat{h}(\hat{w}-\hat{h})[\hat{w}-(1-\eta) \hat{h}]}, \\
\hat{K}_{2}(\hat{x})=-\frac{9(\hat{w}-\hat{h})^{2} \hat{q}_{1}-3 \hat{h}[4 \hat{w}-(4-\eta) \hat{h}] \hat{q}_{2}}{\hat{h}(\hat{w}-\hat{h})^{3}[\hat{w}-(1-\eta) \hat{h}]}-\frac{6(\hat{w}-\hat{h}) \hat{\zeta}_{1}+3[2 \hat{w}-(2-\eta) \hat{h}] \hat{\zeta}_{2}}{(\hat{w}-\hat{h})^{2}[\hat{w}-(1-\eta) \hat{h}]} \\
+\frac{3[4 \hat{w}-(4-\eta) \hat{h}]\left(\hat{\zeta}_{1}-\hat{\zeta}_{2}\right) \tanh \left[\frac{\hat{\kappa}}{2}(\hat{w}-\hat{h})\right]}{\hat{\kappa}(\hat{w}-\hat{h})^{3}[\hat{w}-(1-\eta) \hat{h}]}
\end{gathered}
$$

In the absence of surface tension, the balance of normal stress at the liquid-liquid interface requires that (see details in the appendix) $\hat{p}_{1}=\hat{p}_{2}$, or

$$
\hat{K}_{1}=\hat{K}_{2} \quad \text { at any } \hat{x} \text {. }
$$

From Eqs. (26)-(28), we get after some algebra the following equation

$$
\begin{aligned}
& \left.\hat{\kappa} \eta(1-\eta) \hat{\zeta}_{2} \hat{h}^{5}+\left\{(1-\eta) \hat{q}_{1}+\eta(1-\eta) \hat{q}_{2}+\hat{w} \eta\left[2 \hat{\zeta}_{1}-(1-\eta) \hat{\zeta}\right]\right]\right\} \hat{\kappa} \hat{h}^{4} \\
& -\left[2(2-3 \eta) \hat{q}_{1}-2 \eta \hat{q}_{2}+\hat{w} \eta\left(4 \hat{\zeta}_{1}+\hat{\zeta}_{2}\right)\right] \hat{\kappa} \hat{w} \hat{h}^{3} \\
& +\left[3(2-3 \eta) \hat{q}_{1}-3 \eta \hat{q}_{2}+\hat{w} \eta\left(2 \hat{\zeta}_{1}+\hat{\zeta}_{2}\right)\right] \hat{\kappa} \hat{w}^{2} \hat{h}^{2}-4(1-\eta) \hat{q}_{1} \hat{\kappa} \hat{w}^{3} \hat{h} \\
& +\hat{q}_{1} \hat{\kappa} \hat{w}^{4}+\eta\left(\hat{\zeta}_{1}-\hat{\zeta}_{2}\right)\left[(1-\eta) \hat{h}^{2}+2 \hat{w} \hat{h}-3 \hat{w}^{2}\right] \hat{h}^{2} \tanh \left[\frac{\hat{\kappa}}{2}(\hat{w}-\hat{h})\right]=0 .
\end{aligned}
$$


To maintain continuity of flow through a non-uniform channel, a pressure gradient has to be induced internally. By periodicity, the integral of the induced pressure gradient over one wavelength is zero, however. The net pressure change in one wavelength is solely due to the applied pressure gradient. Hence,

$$
\int_{0}^{1} \hat{K}_{1} \mathrm{~d} \hat{x}=\int_{0}^{1} \hat{K}_{2} \mathrm{~d} \hat{x}=\Delta \hat{P} .
$$

Also by virtue of periodicity, the average displacement of the interface between the two fluids over one wavelength is zero, and the mean position of the interface is the position of the interface when the fluids are static. Hence,

$$
\int_{0}^{1} \hat{h}(\hat{x}) \mathrm{d} \hat{x}=1 .
$$

We may now determine $\hat{h}(\hat{x}), \hat{q}_{1}$ and $\hat{q}_{2}$ by solving Eq. (29) while satisfying the two global conditions (30) and (31). The problem requires the prescription of the following parameters or periodic functions of $\hat{x}: \Delta \hat{P}, \eta, \hat{\kappa}, \hat{\zeta}_{1}, \hat{\zeta}_{2}(\hat{x})$ and $\hat{w}(\hat{x})$. Our solution method is described in Sec. 4.

\section{Special case: flat lower wall with uniform zeta po- tential}

For a flat lower wall $\left(\hat{w}_{0}=0\right)$ and constant wall potential $\hat{\zeta}_{2}$, the interface between the two immiscible fluids is a plane surface and all quantities are independent of the axial coordinate. By substituting $\hat{h}=1, \hat{w}=1+\hat{h}_{0}$ and $\hat{K}_{1}=\hat{K}_{2}=\Delta \hat{P}$ into Eqs. (14), (15) and (20)-(23), we can obtain the following expressions for the velocities:

$$
\begin{aligned}
\hat{u}_{1}=\frac{\eta \hat{y} \Delta \hat{P}}{2\left(\eta+\hat{h}_{0}\right)}\left[\hat{h}_{0}^{2}+(2-\hat{y}) \hat{h}_{0}+\eta(1-\hat{y})\right]-\frac{\eta \hat{y}}{\eta+\hat{h}_{0}}\left(\hat{\zeta}_{1}-\hat{\zeta}_{2}\right) \quad \text { for } 0 \leq \hat{y} \leq 1, \\
\hat{u}_{2}=\frac{\Delta \hat{P}}{2\left(\eta+\hat{h}_{0}\right)}\left(1+\hat{h}_{0}-\hat{y}\right)\left[\eta \hat{y}+(\eta+\hat{y}-1) \hat{h}_{0}\right] \\
+\frac{\left(1+\hat{h}_{0}-\hat{y}\right) \hat{\zeta}_{1}+(\eta+\hat{y}-1) \hat{\zeta}_{2}}{\eta+\hat{h}_{0}}-\hat{\psi} \quad \text { for } 1<\hat{y} \leq 1+\hat{h}_{0}
\end{aligned}
$$


where $\hat{\psi}$ is given by Eq. (6). Integrating the velocities across their respective layers gives the flow rates:

$$
\begin{aligned}
\hat{q}_{1}= & \int_{0}^{1} \hat{u}_{1} \mathrm{~d} \hat{y}=\frac{\eta\left[\left(3 \hat{h}_{0}^{2}+4 \hat{h}_{0}+\eta\right) \Delta \hat{P}-6\left(\hat{\zeta}_{1}-\hat{\zeta}_{2}\right)\right]}{12\left(\eta+\hat{h}_{0}\right)}, \\
\hat{q}_{2}= & \int_{1}^{1+\hat{h}_{0}} \hat{u}_{2} \mathrm{~d} \hat{y} \\
= & \frac{\hat{h}_{0}\left[\hat{h}_{0}\left(\hat{h}_{0}^{2}+4 \eta \hat{h}_{0}+3 \eta\right) \Delta \hat{P}+6 \hat{h}_{0} \hat{\zeta}_{1}+6\left(2 \eta+\hat{h}_{0}\right) \hat{\zeta}_{2}\right]}{12\left(\eta+\hat{h}_{0}\right)} \\
& -\frac{\hat{\zeta}_{1}-\hat{\zeta}_{2}}{\hat{\kappa}} \tanh \left(\frac{1}{2} \hat{\kappa} \hat{h}_{0}\right) .
\end{aligned}
$$

In particular, if no pressure difference is applied externally, i.e., $\Delta \hat{P}=0$, the flow rates reduce to

$$
\begin{gathered}
\hat{q}_{1}=-\eta\left(\hat{\zeta}_{1}-\hat{\zeta}_{2}\right) / 2\left(\eta+\hat{h}_{0}\right) \\
\hat{q}_{2}=\frac{\hat{h}_{0}}{2\left(\eta+\hat{h}_{0}\right)}\left[\hat{h}_{0} \hat{\zeta}_{1}+\left(2 \eta+\hat{h}_{0}\right) \hat{\zeta}_{2}\right]-\frac{\hat{\zeta}_{1}-\hat{\zeta}_{2}}{\hat{\kappa}} \tanh \left(\frac{1}{2} \hat{\kappa} \hat{h}_{0}\right) .
\end{gathered}
$$

\section{Solution method}

For given $\eta, \hat{\kappa}, \hat{\zeta}_{1}, \Delta \hat{P}, \hat{w}(\hat{x})$ and $\hat{\zeta}_{2}(\hat{x})$, the problem is solved when $\hat{h}(\hat{x}), \hat{q}_{i}$ and $\hat{K}_{i}(\hat{x})$ $(i=1,2)$ are found through Eqs. (26), (27), (29), (30) and (31). It is a highly nonlinear system of equations, which can be solved by a trial-and-error numerical scheme. First, the domain $0 \leq \hat{x} \leq 1$ is partitioned into $N-1$ equal intervals such that solutions are to be sought at discrete points $\hat{x}_{i}=(i-1) /(N-1)$ for $i=1, \cdots, N$. Second, with a trial value of $\hat{q}_{2}^{(n)}$, the corresponding values of $\hat{q}_{1}^{(n)}$ and $\hat{h}\left(\hat{x}_{i}\right)$ can be found via Eqs. (29) and (31), where $n$ denotes the number of trials. The pressure gradients at each point $\hat{K}_{1}\left(\hat{x}_{i}\right)$ and $\hat{K}_{2}\left(\hat{x}_{i}\right)$ can then be calculated from Eqs. (26) and (27). Third, the pressure drop over one wavelength $\Delta \hat{P}^{(n)}$ can be evaluated from the integral of $\hat{K}_{1}$ or $\hat{K}_{2}$. This calculated pressure drop $\Delta \hat{P}^{(n)}$ under the trial value of $\hat{q}_{2}^{(n)}$ is compared with the given $\Delta \hat{P}$. If they are sufficiently close to each other, $\hat{q}_{2}^{(n)}$ will be accepted as the solution resulting from the given pressure drop $\Delta \hat{P}$; otherwise, a corrected value of $\hat{q}_{2}$ will be used for the next trial. One possible method to 
estimate $\hat{q}_{2}^{(n+1)}$ is by means of a linear extrapolation:

$$
\hat{q}_{2}^{(n+1)}=\hat{q}_{2}^{(n)}+\frac{\Delta \hat{P}-\Delta \hat{P}^{(n)}}{\Delta \hat{P}^{(n)}-\Delta \hat{P}^{(n-1)}}\left(\hat{q}_{2}^{(n)}-\hat{q}_{2}^{(n-1)}\right) .
$$

This trial-and-error method will proceed until the difference between the calculated and given pressure drop is less than $10^{-5}$.

In the second step described above, with a given value of $\hat{q}_{2}$, the values of $\hat{q}_{1}$ and $\hat{h}\left(\hat{x}_{i}\right)$ are to be obtained from Eqs. (29) and (31). This step itself is also a trial-and-error process. With a guessed value of $\hat{q}_{1}$, the values of $\hat{h}\left(\hat{x}_{i}\right)$ can be found by solving Eq. (29) numerically. The integral of $\hat{h}\left(\hat{x}_{i}\right)$ over one wavelength is then computed in order to check whether Eq. (31) is satisfied. If not satisfied, a new trial will be conducted. This trial-and-error process will go

on until the integral of $\hat{h}$ over one wavelength is sufficiently close to unity, say $\left|\int_{0}^{1} \hat{h} \mathrm{~d} x-1\right| \leq$ $10^{-5}$.

\section{Results and discussion}

For simplicity, we shall from here on omit the overhead carets in our notation. Let us introduce the following sinusoidal functions of $x$ for the lower wall topography and the zeta potential on the lower wall:

$$
\begin{gathered}
w(x)=1+h_{0}+w_{0}[1-\cos (2 \pi x)], \\
\zeta_{2}(x)=\bar{\zeta}_{2}+\zeta_{2}^{\prime} \cos (2 \pi x+\theta),
\end{gathered}
$$

where, as shown in Fig. 1, $h_{0}$ is the height of the mean position of the interface above the crest of the bottom topography, $w_{0}$ is the amplitude of the wavy form of the lower wall $\left(2 w_{0}\right.$ being the depth of the groove), $\bar{\zeta}_{2}$ and $\zeta_{2}^{\prime}$ are the average and amplitude of the varying zeta potential, respectively, and $\theta$ is the phase shift between the two distributions. Although idealized, these sinusoidal waveforms can provide results revealing the physics involved in the present problem.

As shown in Fig. 1, a conducting fluid (denoted by subscript "2") lying atop the bottom wavy wall located at $y=w(x)$ is simultaneously pumped by hydrodynamic and electric 
forcings, while an upper fluid (denoted by subscript "1") of low conductivity is driven into motion by the hydrodynamic forcing as well as viscous drag on the interface $y=h(x)$. The hydrodynamic forcing acting on both fluids is due to an induced pressure gradient in combination with an externally applied pressure difference $\Delta P$. The variations of the bottom topography and wall potential will interact with each other, resulting in an enhanced or weakened induced pressure gradient, which will influence the flow field directly. The effects of these two variations, one geometric and one electrokinetic, on the flow field of this two-fluid system are examined here. Other factors, including the hydrodynamic forcing $\Delta P$, the fluid height $h_{0}$, the viscosity ratio between the two fluids $\eta$, and the interfacial zeta potential $\zeta_{1}$, are also investigated. In the following discussions, the Debye parameter $\kappa$ is taken to be a large value, say $\kappa=100$, to ensure that the EDLs are much thinner than the fluid layers.

Let us first look into the particular case of a flat interface. This is possible only when the flow is purely unidirectional without any non-uniformity in the axial direction, namely, $w \equiv 1+h_{0}$ and $\zeta_{2} \equiv \bar{\zeta}_{2}$. The axial velocity profiles for $\Delta P=0,1$ are shown in Figs. 2(a, b), respectively. As thin EDLs are assumed, the electric forcing is confined to narrow regions in the vicinity of the lower wall and the interface, while the electrokinetic effect is negligible in the interior. As shown in Fig. 2(a) where $\Delta P=0$, the velocity develops a sharp gradient within the thin EDLs and has a linear profile outside the EDLs. In the case of equal zeta potentials on the wall and at the interface $\left(\zeta_{1}=\zeta_{2}\right)$, the upper layer does not move as if it were a static rigid body since the net shear stress acting on the interface is zero (or an exact balance between the viscous and Maxwell shear stresses). The free charge in the EDL near the interface gives rise to an interfacial zeta potential and the so-called Maxwell stress on the liquid-liquid interface. This interfacial electro-stress introduces a stress-jump across the liquid-liquid interface, as described by Eq. (19), where the component of electro-stress tangential to the interface is given by

$$
\left.\eta \frac{\partial \psi}{\partial y}\right|_{y=h}=-\frac{\eta \kappa \zeta_{1}}{\tanh [\kappa(w-h)]}+\frac{\eta \kappa \zeta_{2}}{\sinh [\kappa(w-h)]},
$$

of which the second term is negligible compared with the first term for $\kappa(w-h) \gg 1$. If the interfacial zeta potential is zero, i.e., $\zeta_{1}=0$, viscous stress will be continuous across the liquid-liquid interface, and in the absence of an interfacial EDL the velocity has a smooth profile across the two layers for $\eta=1$. For a positive interfacial zeta potential, the minus sign 
in Eq. (41) implies that the tangential viscous and electro-stresses act in opposite directions to each other, which leads to a reduced net shear stress on the upper fluid. This is illustrated by the results shown in Figs. 2(a, b) for $\zeta_{1}=0.5,1$, where the velocity gradient is much milder in the upper layer than that in the lower layer near the liquid-liquid interface. In addition, as in Eq. (41), the electro-shear stress is linearly proportional to the interfacial zeta potential. The stress, a negative quantity, becomes larger in magnitude with larger $\zeta_{1}$. As has been discussed above, the velocity in the upper layer will be identically zero when $\zeta_{1}=1$.
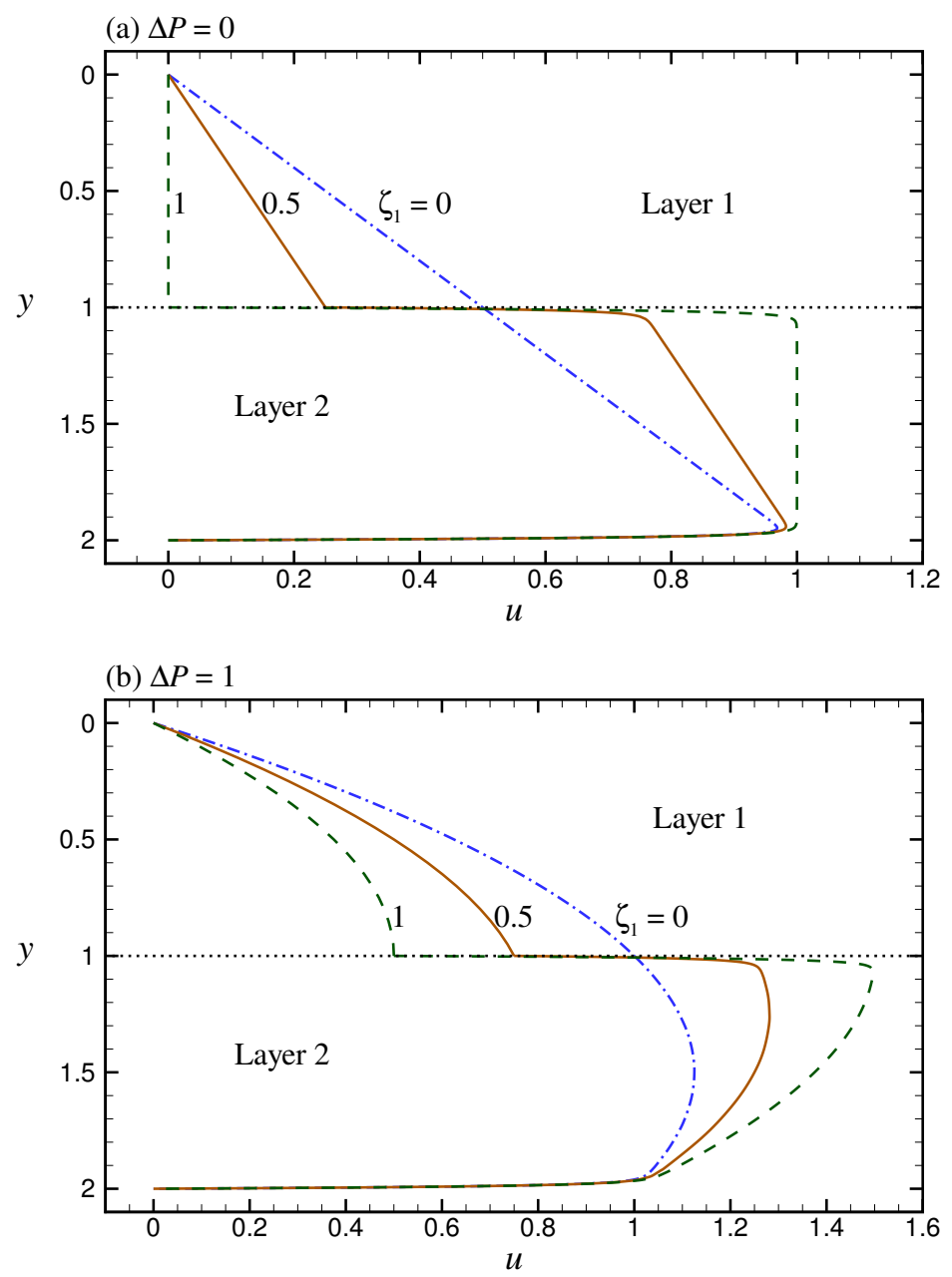

Figure 2: Axial velocity profile, $u(y)$, for flow in a uniform channel under (a) $\Delta P=0$, (b) $\Delta P=1$, and $\zeta_{1}=0,0.5,1$, where $\eta=1, \zeta_{2}=1$ and $h_{0}=1$. The dotted line at $y=1$ denotes the interface between the non-conducting (layer 1) and conducting (layer 2) fluids.

Flow in a non-uniform channel is characterized by an induced pressure gradient and the deformation of the liquid-liquid interface. The interfacial zeta potential will play a central role 
in determining the pressure gradient and the resulting flow rates. For $\Delta P=0$, the flow rates of the two fluids as functions of $\zeta_{1}$ are shown in Fig. 3(a), and the velocity profiles $u(x=0, y)$ and the pressure gradients $K(x)$ for different interfacial zeta potentials, $\zeta_{1}=0,0.5,1$, are shown in Figs. 3(b, c), respectively. From Fig. 3(a), it is seen that as $\zeta_{1}$ increases, the flow rate decreases for the non-conducting fluid, while increases for the conducting fluid. These opposite trends are similar to those seen above for the case of a uniform channel. As the interfacial zeta potential increases, the viscous drag applied by the conducting fluid on the non-conducting fluid is weakened, resulting in a smaller flow rate of the latter. This effect is further revealed in Fig. 3(b). Increasing the interfacial zeta potential will weaken the flow of the non-conducting fluid, but enhance the flow of the conducting fluid. As expected, the interfacial zeta potential will also influence the pressure gradient; see Fig. 3(c). Increasing the interfacial zeta potential will in general lower the magnitude of the pressure gradient.

The velocity profiles shown in Fig. 3(b) reveal that the velocity is negative near the upper wall. Such a reversed current suggests a recirculation zone in the upper layer. Figures 4(ac) show the corresponding two-dimensional flow fields. Even for $\zeta_{1}=0$, recirculation rolls occur in the non-conducting fluid layer, which are attributable to the interaction between the non-uniformities in the zeta potential and the shape of the lower wall. These recirculation cells develop at the narrowest cross-section of the channel $(x=0$ or 1$)$, where the induced pressure gradient is the maximum negative, as shown in Fig. 3(c). The adverse pressure gradient outweighs the favorable viscous drag at the liquid-liquid interface and causes the flow to alter its direction near the top of the channel. As $\zeta_{1}$ increases, the recirculation cells will grow in size such that they tend to extend across most of the upper layer. Owing to the recirculation cells, the interface is displaced downward near $x=0$ and $x=1$, and upward near $x=0.5$. The deformation of the interface is, however, not significantly affected by $\zeta_{1}$ albeit the increase of $\zeta_{1}$ leads to bigger recirculation cells. Figure 4(d) shows the extremum positions of the interface, $h_{\min }$ at $x=0.5$ and $h_{\max }$ at $x=0$ or 1 , as functions of $\zeta_{1}$. For example, when the interfacial zeta potential $\zeta_{1}$ increases from 0 to $1, h_{\max }$ only changes modestly from 1.196 to 1.173 , despite the growth of the recirculation cells.

We next show in Fig. 5 the effects of the phase shift $\theta$ on the flow field. First, Fig. 5(a) shows how the flow rates of two fluids may change depending on $\theta$. For either the conducting 


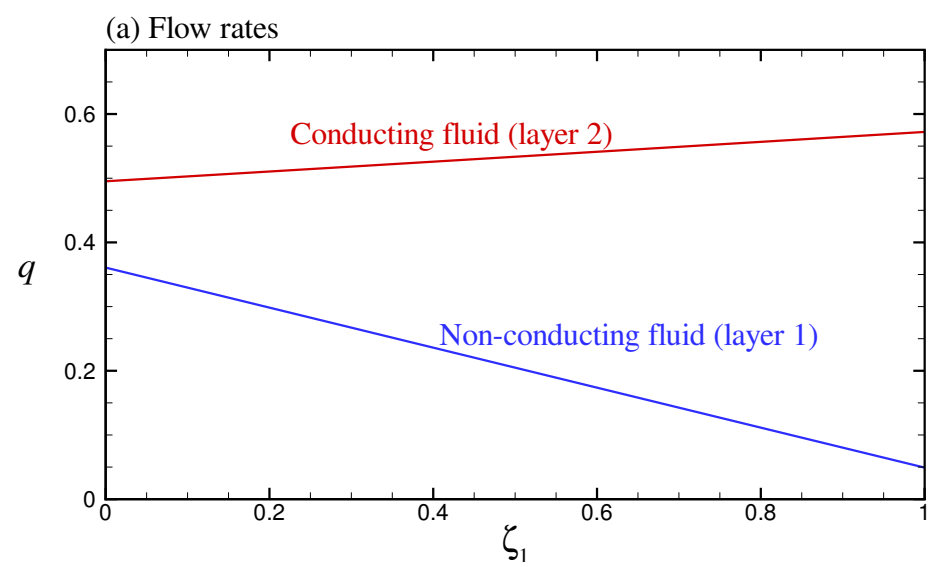

(b) Velocity profile at $x=0$

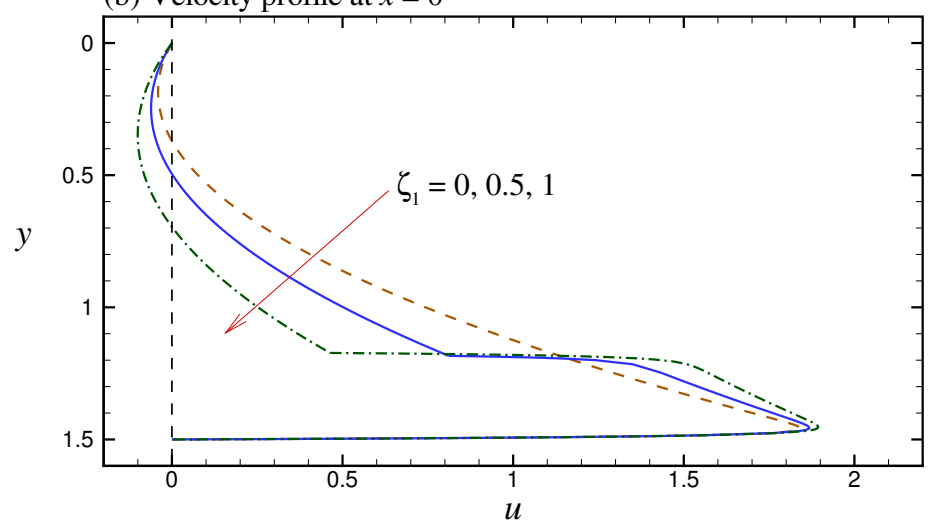

(c) Pressure gradient distribution

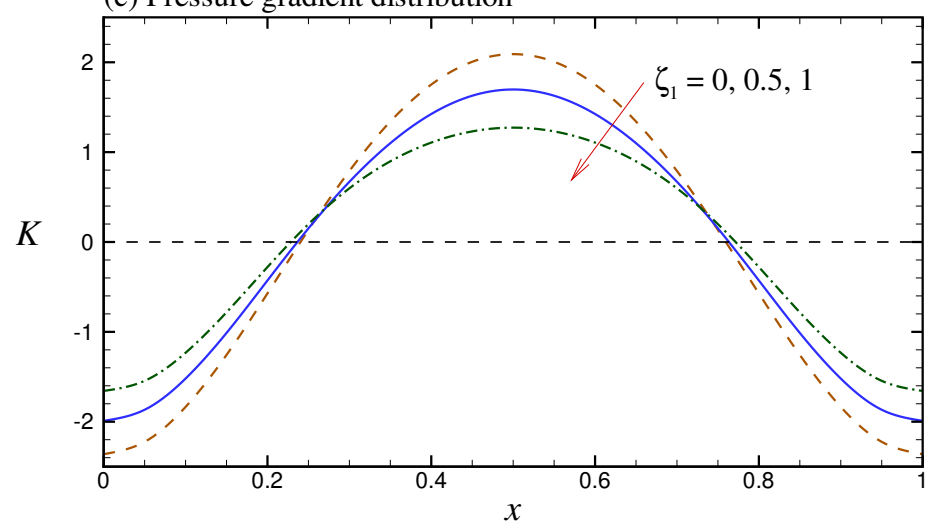

Figure 3: (a) Flow rates of the two fluids as functions of the interfacial zeta potential $\zeta_{1}$; (b) axial velocity profile at the narrowest cross-section, $u(x=0, y)$, for $\zeta_{1}=0,0.5,1$; and (c) pressure gradient distribution, $K(x)$, for $\zeta_{1}=0,0.5,1$, where $\Delta P=0, \eta=1, h_{0}=0.5$, $w_{0}=0.1, \bar{\zeta}_{2}=\zeta_{2}^{\prime}=1$ and $\theta=0$. 


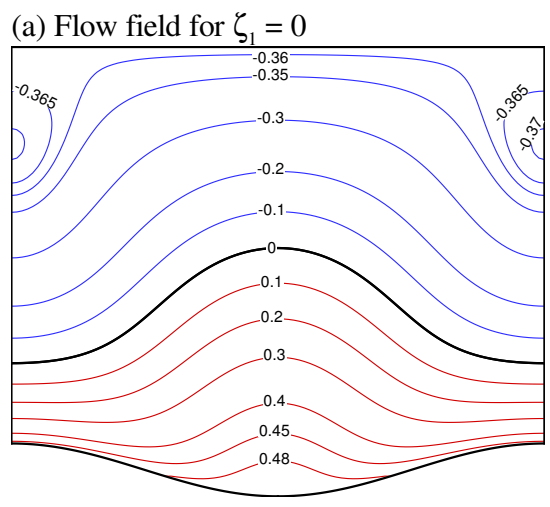

(b) Flow field for $\zeta_{1}=0.5$

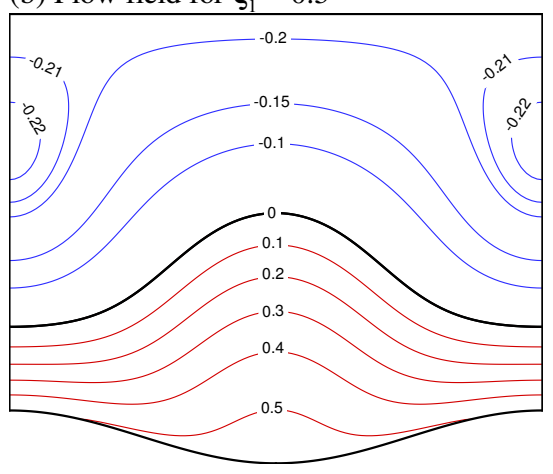

(c) Flow field for $\zeta_{1}=1$
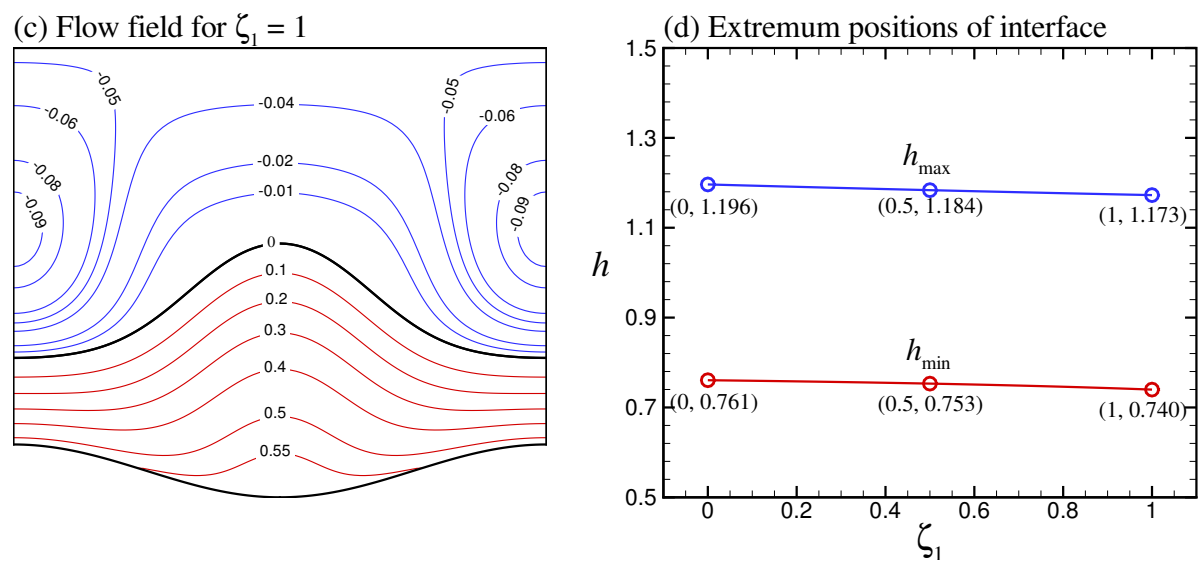

Figure 4: Flow field with streamlines for (a) $\zeta_{1}=0$, (b) $\zeta_{1}=0.5$, and (c) $\zeta_{1}=1$, and (d) the extremum positions of the interface, $h_{\min }, h_{\max }$, as functions of $\zeta_{1}$, where other parameters have the same values as those for Fig. 3. The streamline with the zero value is the interface between the two fluids. 
or non-conducting fluids, as $\theta$ increases from 0 to $2 \pi$, the flow rate first decreases from the maximum at $\theta=0$ to the minimum at $\theta=\pi$, and then increases back to the maximum at $\theta=2 \pi$. We can reason that the phase $\theta=0$ corresponds to the case where the maximum zeta potential $\zeta_{2}$ (hence the strongest point of electrokinetic pumping) is located at the narrowest section of the channel. The resulting flow rate is therefore the maximum. In contrast, the phase $\theta=\pi$ corresponds to the case where the minimum zeta potential $\zeta_{2}$ (hence the weakest point of electrokinetic pumping) is located at the narrowest section of the channel. The resulting flow rate is therefore the minimum. This effect is well known in the literature: flow in a non-uniform channel is essentially rate-limited by the conditions prevailing at the narrowest cross-section of the channel. Let us examine in further detail the flow under different values of $\theta$. Figure 5(b) shows the deformed shape of the interface for $\theta=0, \pi / 4$, $\pi / 2,3 \pi / 4$ and $\pi$. For $\theta=0$, the interface is relatively flat, slightly undulating about the mean position of $y=1$. As the phase shift increases in the range $0 \leq \theta \leq \pi$, the displacement of the interface is amplified, resulting in a higher crest and a lower trough. The flow fields for $\theta=0$ and $\theta=\pi$ are shown in Figs. 5(c,d), respectively. While the flow is non-recirculating for $\theta=0$, a large recirculation cell emerges occupying much of the upper layer for $\theta=\pi$. This explains why the flow rate of the non-conducting fluid is nearly zero when $\theta=\pi$, as shown in Fig. 5(a). For fluid transport, the occurrence of recirculation cells is not desirable, and the phase shift $\theta=\pi$ should be avoided. Let us recall the interfacial displacement shown in Fig. 5(b). Increasing $\theta$ from zero will cause a recirculation cell to appear in the upper fluid, as reflected by the enhanced downward displacement of the interface. The center of the recirculating cell migrates from right to left as $\theta$ increases. As the cell shifts toward the middle, the restraining effect of the upper wall on the formation of the recirculation is weakened, and therefore the cell will grow in size. This also explains why the interfacial displacement is larger for a larger phase shift.

The amplitude $w_{0}$ of the wavy shape of the lower wall is one of parameters for the present problem. Its effects can been seen from Fig. 6. In Figs. 6(a, b), we first show the flow rates of the two fluids for different viscosity ratios as functions of $w_{0}$. For a smaller viscosity ratio $\eta$, or a more viscous non-conducting fluid, the flow rate $q_{1}$ is smaller, which is expected. As the amplitude increases, $q_{1}$ first increases and then decreases. There exists an optimum 

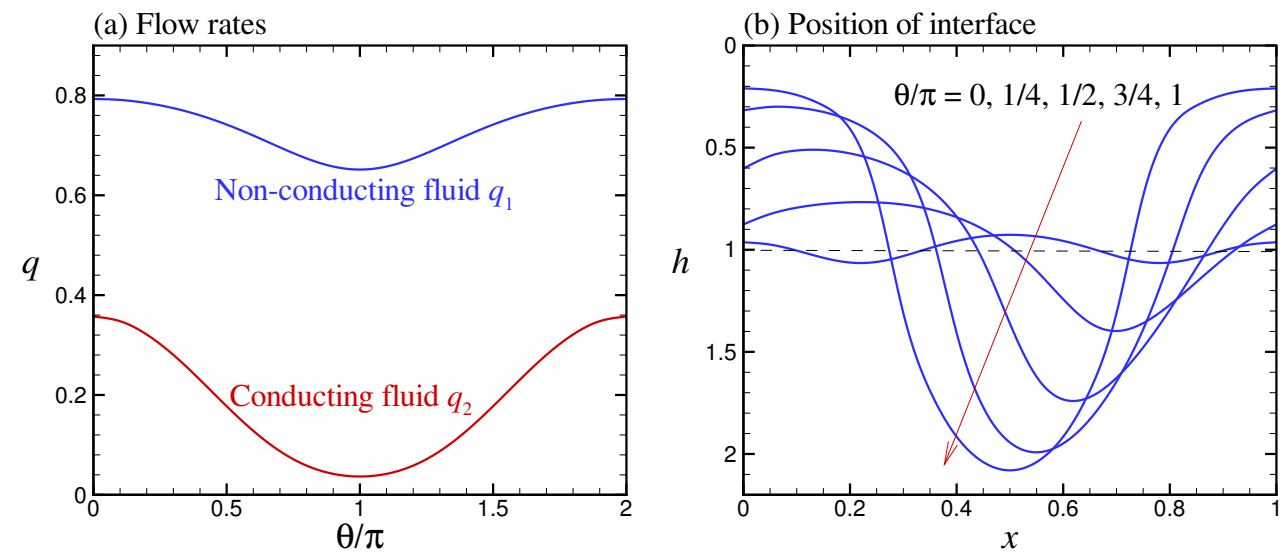

(c) Flow field for $\theta=0$

(d) Flow field for $\theta=\pi$
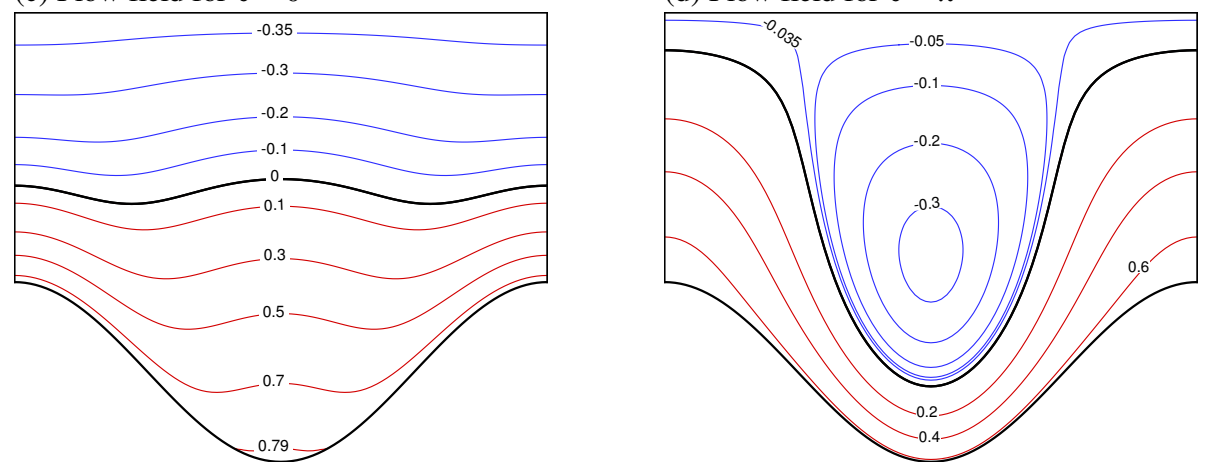

Figure 5: (a) Flow rates $q$ of the two fluids as functions of the phase shift $\theta$; (b) position of the liquid-liquid interface for $\theta / \pi=0,1 / 4,1 / 2,3 / 4,1$; (c) flow field with streamlines for $\theta=0$; (d) flow field with streamlines for $\theta=\pi$, where $\Delta P=0, h_{0}=w_{0}=0.5, \eta=1$, $\bar{\zeta}_{2}=\zeta_{2}^{\prime}=1$ and $\zeta_{1}=0$. The streamline with the zero value is the interface between the two fluids. 
amplitude, denoted by $w_{0 \text { opt }}$, which gives rise to maximum flow rate of the non-conducting fluid, denoted $q_{1 \max }$. These two quantities as functions of the viscosity ratio $\eta$ are shown in Figs. 6(e,f) (solid lines), where for comparison the flow rate $q_{1}$ corresponding to $w_{0}=0$ (dashed line) is also presented. For EO flow of a single fluid, it has been found by Ajdari [19,20] that wall undulations, in combination with modulated wall potential, may contribute to a net flow even when the wall potential has a zero mean. For EO flow of two immiscible fluids, the wall undulation can likewise enhance the flow rate when interacting with nonuniformly distributed zeta potentials. Figures $6(\mathrm{c}, \mathrm{d})$, which show the the pressure gradient and the interfacial displacement over one wavelength, help explain why there may exist an optimum amplitude of the wall undulation for maximum flow rate. By continuity, the flow rate is independent of axial position, so we may consider flow through any cross-section, such as $x=0$. For given $\zeta_{1}$, the flow rate of the non-conducting fluid is determined by the hydrodynamic forcing (i.e., the pressure gradient) and the thickness of the fluid layer (or the position of the interface). Either a larger pressure gradient or a thicker fluid layer will lead to a larger flow rate. As is shown in Figs. 6(c, d), increasing the amplitude $w_{0}$ will increase the pressure gradient at $x=0$ but decrease the thickness of the non-conducting fluid layer at this axial position. These two competing effects will counteract each other such that the flow rate may attain a maximum at a certain value of $w_{0}$. For the conducting fluid, an upward displacement of the interface means a thicker fluid layer, and therefore the flow rate $q_{2}$ will increase monotonically with increasing $w_{0}$. From Fig. 6(f), we may see that the maximum possible increase in the flow rate of the non-conducting fluid, which can be achieved by a wall amplitude equal to the optimum value, is more significant for larger $\eta$, or a non-conducting fluid of lower viscosity.

It is of interest to look into the interaction between variations of the wall potential and wall shape if the wall is on average electro-neutral, i.e., $\bar{\zeta}_{2}=0$. For a single fluid, Ajdari [20] has shown that periodic variation of a zeta potential that has a zero mean can produce only convective cells but not a net flow. However, if a wall is non-uniform in both shape and potential distribution, a net flow can happen when the symmetry is broken. Let us here investigate similar interacting effects for the present two-fluid problem. Figures $7(\mathrm{a}-\mathrm{c})$ show the flow fields for $w_{0}=0.2,0.5$ and 1 , where both the applied pressure gradient and average 

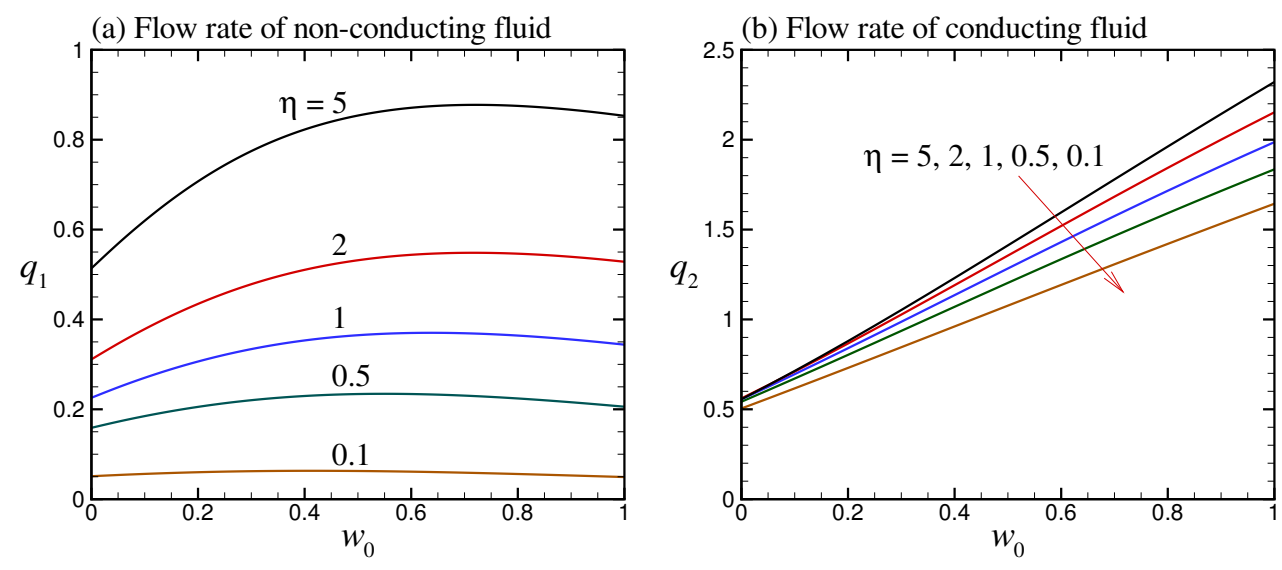

(c) Pressure gradient distribution
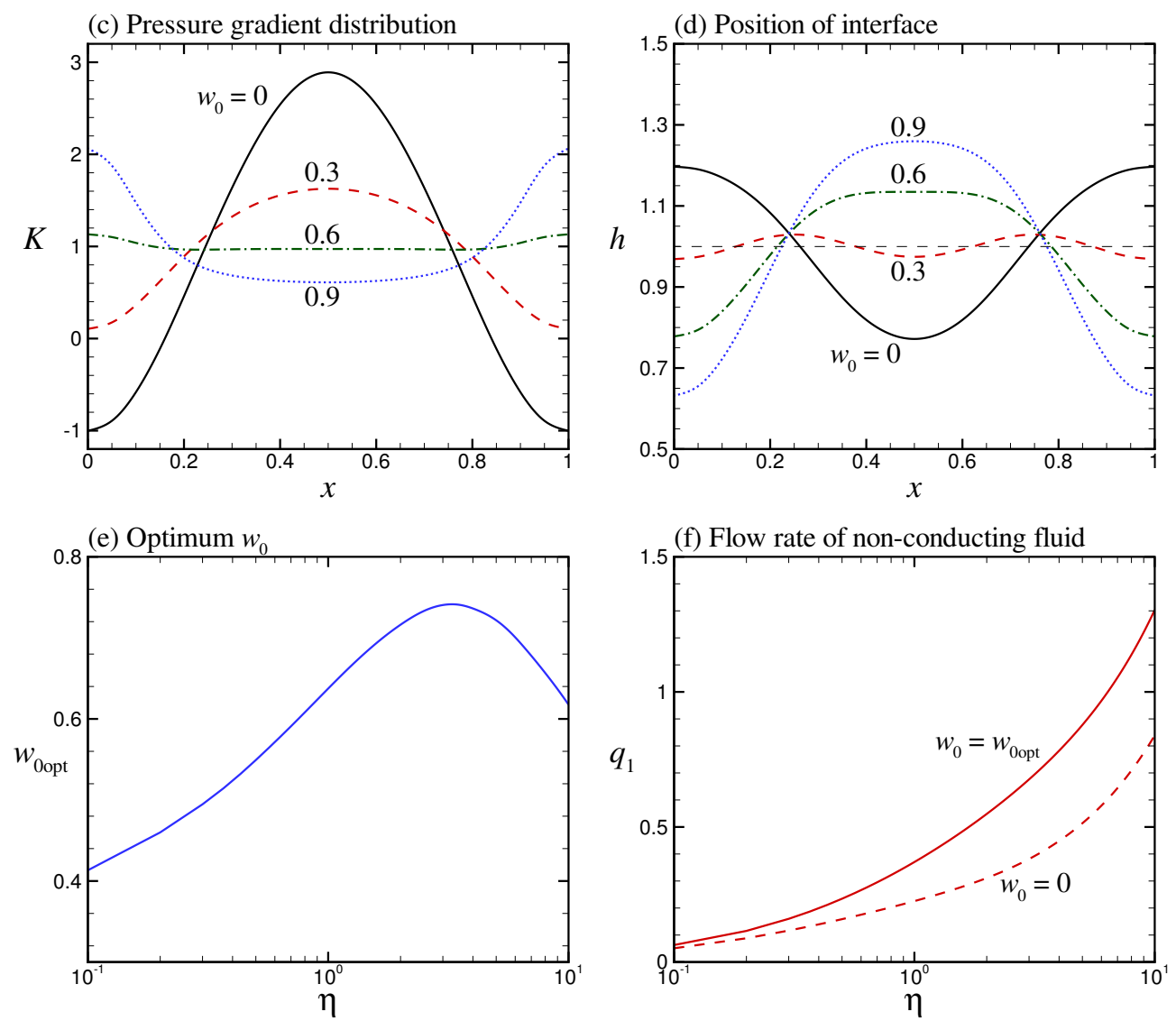

Figure 6: Flow rates (a) $q_{1}$, and (b) $q_{2}$ as functions of the amplitude of the wall undulation $w_{0}$ for $\eta=0.1,0.5,1,2,5$, where $\Delta P=1, h_{0}=0.5, \zeta_{1}=\bar{\zeta}_{2}=\zeta_{2}^{\prime}=1$ and $\theta=0$; (c) pressure gradient distribution, $K(x)$, and (d) position of the interface, $h(x)$, for $w_{0}=0,0.3,0.6,0.9$ for $\eta=1$, where other parameters have the same values as those given in (a,b); (e) optimum value of $w_{0}$, and (f) corresponding maximum flow rate $q_{1}$ (solid line) as functions of $\eta$, where the dashed line in (f) is for the flow rate $q_{1}$ corresponding to $w_{0}=0$. 
zeta potentials are zero. It is interesting to find that recirculation cells appear not only in the non-conducting fluid, but also in the conducting fluid. These recirculating cells are resulting from the counter-action between the hydrodynamic and electric forcings, provided that the induced pressure gradient/potential is maximum negative/positive at $x=0$ or $x=1$ and maximum positive/negative at $x=0.5$. For the non-conducting fluid, the recirculation is centered at $x=0$ and $x=1$, while for the conducting fluid the recirculation is centered at $x=0.5$. These recirculation cells are thereby distributed in a staggered manner along the channel, resulting in some strong upward and downward displacements of the liquid-liquid interface, especially for small $w_{0}$ as shown in Fig. $7(\mathrm{a})$. We note that for $w_{0}=0.2$, the interface is so strongly deformed that it nearly touches the lower wall at some positions (e.g., $\left.\left.(w-h)\right|_{x=0}=0.0288\right)$, leaving a very thin fluid layer of forward flow at these positions. Such a locally very thin lower layer suggests that, as $w_{0}$ reduces to zero, the net flow of the lower layer also reduces to zero when the layer is no longer continuous but virtually split into isolated recirculating cells. Nevertheless, the net flow of the upper layer may remain finite even when that of the lower layer vanishes. Figure $7(d)$ shows how the flow rates increase with increasing $w_{0}$.

The height $h_{0}$ of the conducting fluid above the crest of the lower wall is another parameter for the present problem. For EO flow of two fluids in a uniform channel, Afonso et al. [39] concluded that this height of the conducting fluid should be kept small in order to obtain a higher flow rate of the non-conducting fluid. However, they only examined the case where the externally applied pressure gradient is zero. For a plane interface, which occurs when the channel is strictly uniform, i.e., flat channel walls and uniform zeta potentials, Eq. (34) gives the analytical expression for the flow rate of the non-conducting fluid, $q_{1}$. In order to determine the relationship between $q_{1}$ and the height of the conducting fluid $h_{0}$, we take the derivative of Eq. (34) with respect to $h_{0}$, leading to the following result:

$$
\frac{\partial q_{1}}{\partial h_{0}}=\frac{\eta}{4\left(\eta+h_{0}\right)^{2}}\left[\left(h_{0}^{2}+2 \eta h_{0}+\eta\right) \Delta P+2\left(\zeta_{1}-\zeta_{2}\right)\right] .
$$

From the above equation, it is obvious that in addition to the potentials, the derivative $\partial q_{1} / \partial h_{0}$ is also affected by $\eta$ and $h_{0}$. We show $\partial q_{1} / \partial h_{0}$ as a function of $h_{0}$ for different $\Delta P$ and $\eta$ in Figs. 8(a, b), respectively. In Fig. 8(a), $\partial q_{1} / \partial h_{0}$ is always negative for $\Delta P=0$ within the given range of $h_{0}$. This implies that $q_{1}$ will increase monotonically as $h_{0}$ decreases. 
(a) Flow field for $w_{0}=0.2$

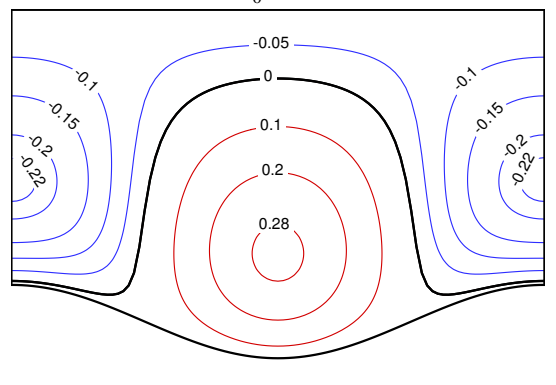

(b) Flow field for $w_{0}=0.5$
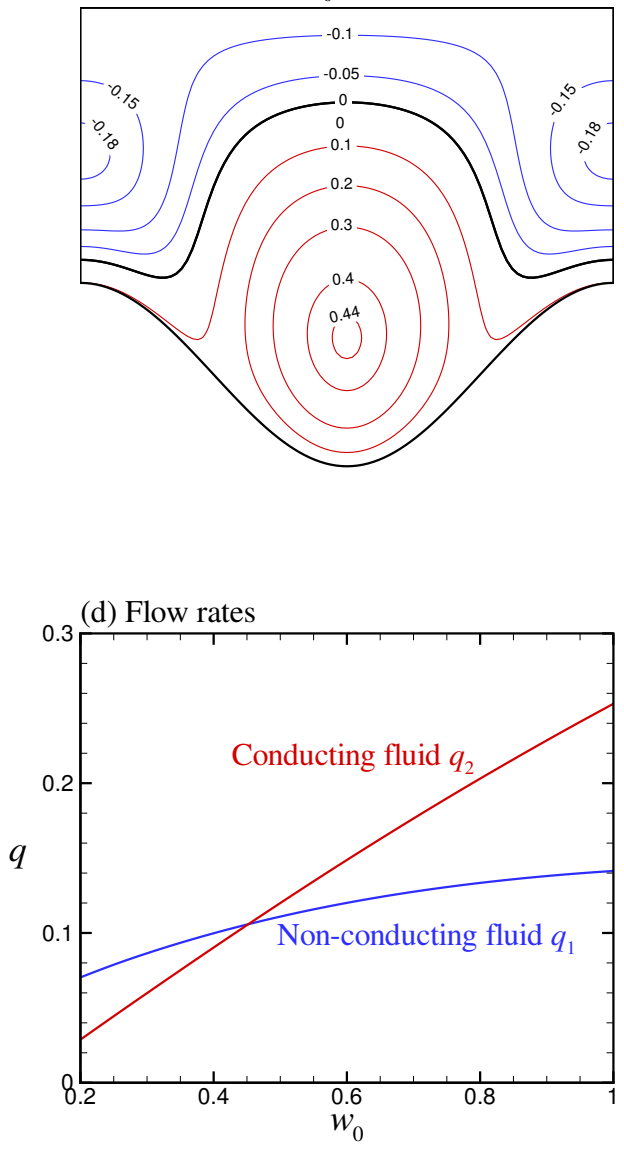

(c) Flow field for $w_{0}=1$

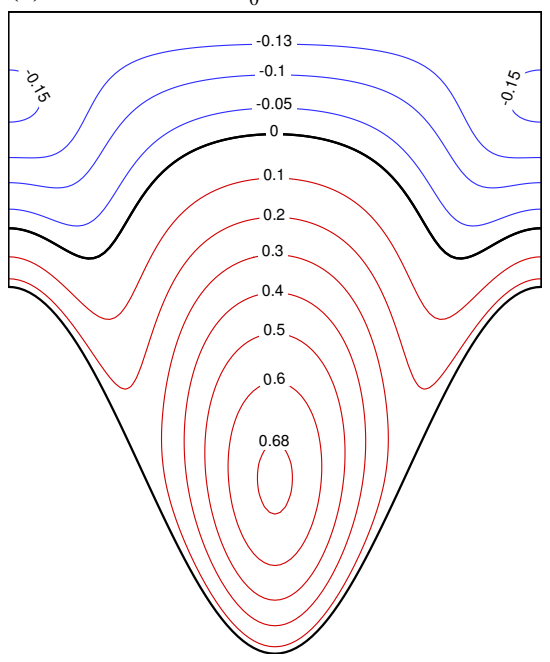

Figure 7: Flow fields with streamlines for (a) $w_{0}=0.2$, (b) $w_{0}=0.5$, (c) $w_{0}=1$; (d) flow rates of the two fluids as functions of $w_{0}$, where $\Delta P=0, \eta=1, h_{0}=0.5, \zeta_{1}=\bar{\zeta}_{2}=0, \zeta_{2}^{\prime}=1$ and $\theta=0$. The streamline with the zero value is the interface between the two fluids. 
As a result, the value of $h_{0}$ has to be kept small in order to obtain large $q_{1}$, which is consistent with the finding of Afonso et al [39]. However, for $\Delta P=1$ or 2 , a larger $h_{0}$ is needed in order to generate a larger volume flow rate. A similar trend can be seen in Fig. 8(b), where different values of $\eta$ may lead to distinct relationships between $q_{1}$ and $h_{0}$. From Figs. 8(c, d), we may further infer that the flow rate $q_{1}$ can be affected in different manners by the height $h_{0}$, depending on the amplitudes $w_{0}$ and $\zeta_{2}^{\prime}$ of the wall undulation and potential modulation.
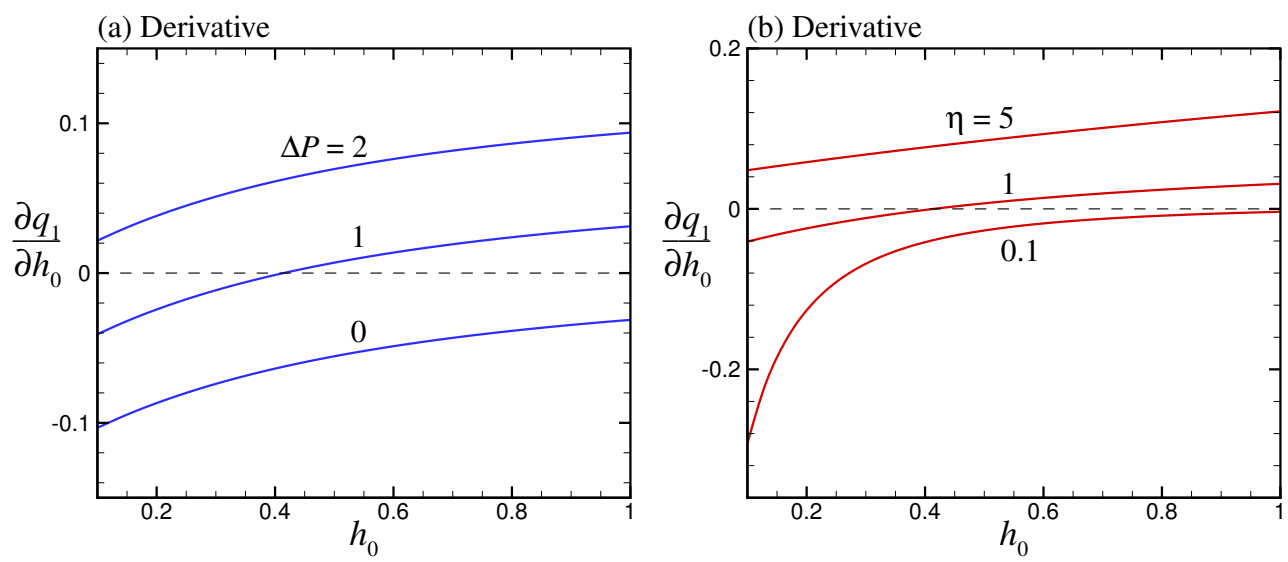

(c) Flow rate of non-conducting fluid
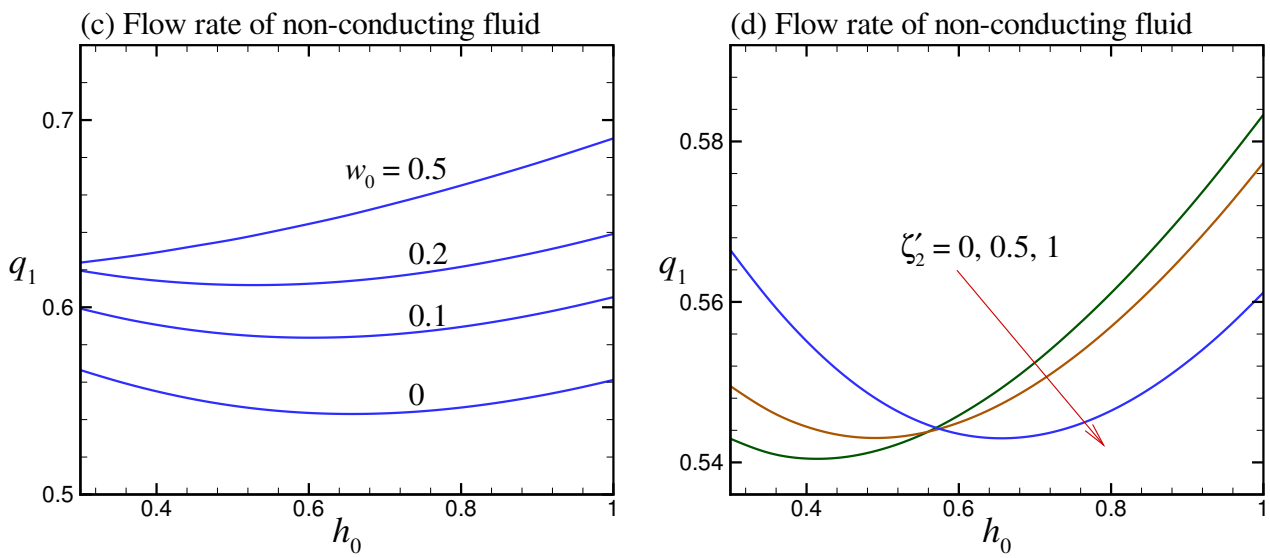

Figure 8: For flow in a uniform channel, the derivative $\partial q_{1} / \partial h_{0}$ as a function of $h_{0}$ for (a) $\Delta P=0,1,2$, and $\eta=1$, and (b) $\Delta P=1$, and $\eta=0.1,1,5$, where $\zeta_{1}=0$ and $\zeta_{2}=1$. For flow in a non-uniform channel, flow rate $q_{1}$ as a function of $h_{0}$ for (c) $w_{0}=0,0.1,0.2,0.5$ and $\zeta_{2}^{\prime}=1$, and $(\mathrm{d}) w_{0}=0$ and $\zeta_{2}^{\prime}=0,0.5,1$, where $\Delta P=1, \eta=1, \zeta_{1}=0, \bar{\zeta}_{2}=1$ and $\theta=0$. 


\section{Concluding remarks}

Electrokinetics-based micropumps cannot be applied directly to non-polar aqueous media with very low ionic conductivity, such as oil, ethanol and organic solvents. To circumvent this shortcoming of electroosmotic (EO) pumping, a novel two-layer system has been proposed: using a near-wall conducting fluid that moves under EO forcing to pull an interior nonconducting medium through viscous action on the fluid-fluid interface. In order to find out how non-uniform wall patterns may affect the flow of the two fluids, we have accomplished a theoretical study on two-fluid electroosmotic flow in a slit microchannel with gradually varying wall shape and potential. The two-fluid system under consideration is composed of one non-conducting working fluid and a conducting sheath fluid, being separated by a sharp interface that may deform depending on the local kinematic and dynamic conditions on the interface. An electric double layer develops in the conducting fluid near the fluid-fluid interface, and also near the lower wall. Both viscous and Maxwell stresses are taken into account in the balance of shear stresses along the interface between the two fluids. Under the combined action of pressure gradient and electric forcing, the conducting sheath fluid is actuated and is used to drag the working fluid into motion. The varying shape and electric potential modulation on the lower wall are assumed to be periodic functions of axial position, while the upper wall is assumed to be a flat surface. Owing to the non-uniformities in the shape and potential on the lower wall, the interface between the two immiscible fluids

will deform and a pressure gradient is internally induced in order to maintain continuity of flow along the channel. On the basis of lubrication approximation, we have deduced formal expressions for the potential in the conducting fluid and the axial flow velocities of the two fluids. The displacement of the interface and the induced pressure gradient, which are periodic functions of zero average, are determined by means of a trial-and-error numerical scheme.

The interfacial zeta potential, determined by the ionic properties of two fluids, $\mathrm{pH}$ values and concentrations of electrolytes, is found to have possibly significant effect on the flow field. If the wall is patterned with alternating positive-negative surface potentials, recirculating cells will develop in the two fluids, which may cause the interface to deform appreciably. As the interfacial zeta potential increases, these cells grow in size but the deformation is not significantly affected by this potential. We have also examined the effects of the phase shift 
between the wall undulation and the potential modulation. The flow rates of both conducting and non-conducting fluids are maximum/minimum when these two wall patterns are inphase/ $\pi$-out-of-phase with each other. Also, the displacement of the interface is amplified by increasing the phase shift. In the presence of wall potential modulation, at any crosssection, increasing the amplitude of the wall undulation may increase the induced pressure gradient on the one hand but decrease the thickness of the non-conducting fluid layer on the other hand. Because of these two opposite effects, there exists an optimum amplitude of the wall undulation that gives rise to maximum flow rate of the non-conducting fluid. At this optimum amplitude, the flow rate of the non-conducting fluid is much enhanced compared with the counterpart for zero amplitude. The interaction between the wall undulation and the modulated potential can be utilized to improve the efficiency of a two-fluid EO pumping. The findings of the present study may help one to look for a suitable configuration for the optimum performance of a two-fluid electrokinetics-based micropump.

We finally note that many other effects of non-uniform wall patterns on two-fluid EO flow remain unknown and deserve to be examined in a future study. If the wall patterns, such as zeta potential and wall shape, are of different wavelengths or may even be non-periodic, it is possible that the enhancement of volumetric flux due to their interactions is dramatically different from what have been found here. A more comprehensive future study incorporating these and other geometrical and electrokinetic factors will enable us to have a more thorough understanding about the performance of a two-fluid EO system.

\section{Acknowledgments}

The authors are very grateful to the two anonymous reviewers for their comments, which have helped improve the writing of this paper. Financial support by the Research Grants Council of the Hong Kong Special Administrative Region, China, through General Research Fund Project No. 17206615 is also gratefully acknowledged. 


\section{Appendix}

This appendix is to explain how boundary conditions are developed from the balance of shear and normal stresses at the liquid-liquid interface.

The stress tensor $\boldsymbol{\tau}$, incorporating the components arising from hydrodynamic stress and Maxwell stress, is expressed in terms of the velocity $\vec{u}$ and the electric field $\vec{E}$,

$$
\boldsymbol{\tau}=\mu\left[\vec{\nabla} \vec{u}+(\vec{\nabla} \vec{u})^{\mathrm{T}}\right]+\epsilon\left[\vec{E} \vec{E}-\frac{1}{2}(\vec{E} \cdot \vec{E}) \boldsymbol{I}\right]
$$

in which $\mu$ and $\epsilon$ are the dynamic viscosity and the permittivity of the liquid, and $\boldsymbol{I}$ is the unit tensor. On the right-hand side of the above equation, the first and second terms represent the viscous stress $\boldsymbol{\tau}_{h}$ and the Maxwell stress $\boldsymbol{\tau}_{e}$, respectively. For a two-dimensional flow in a Cartesian system $(x, y)$, these two contributions can be written by their component form as follows,

$$
\begin{gathered}
\boldsymbol{\tau}_{h}=\mu\left(\begin{array}{cc}
2 \frac{\partial u}{\partial x} & \frac{\partial u}{\partial y}+\frac{\partial v}{\partial x} \\
\frac{\partial v}{\partial x}+\frac{\partial u}{\partial y} & 2 \frac{\partial v}{\partial y}
\end{array}\right), \\
\boldsymbol{\tau}_{e}=\epsilon\left(\begin{array}{cc}
E_{x}^{2}-\frac{1}{2} E^{2} & E_{x} E_{y} \\
E_{y} E_{x} & E_{y}^{2}-\frac{1}{2} E^{2}
\end{array}\right),
\end{gathered}
$$

in which

$$
E_{y}=-\frac{\partial \psi}{\partial y} \quad \text { and } \quad E^{2}=E_{x}^{2}+E_{y}^{2}
$$

On the interface between the two immiscible fluids (denoted by subscripts "1" and "2"), which is a nearly plane surface, the conditions of the balance of shear and normal stresses can be expressed as follows:

$$
\begin{gathered}
\left(\boldsymbol{\tau}_{1} \cdot \vec{e}_{y}\right) \cdot \vec{e}_{x}=\left(\boldsymbol{\tau}_{2} \cdot \vec{e}_{y}\right) \cdot \vec{e}_{x} \\
p_{1}-p_{2}+\left[\left(\boldsymbol{\tau}_{2}-\boldsymbol{\tau}_{1}\right) \cdot \vec{e}_{y}\right] \cdot \vec{e}_{y}=0
\end{gathered}
$$

where $\left(\vec{e}_{x}, \vec{e}_{y}\right)$ are the unit vectors in the $x$ - and $y$-directions. Substituting the stress components, Eqs. (47) and (48) can be written as

$$
\begin{gathered}
\mu_{1}\left(\frac{\partial u_{1}}{\partial y}+\frac{\partial v_{1}}{\partial x}\right)=\mu_{2}\left(\frac{\partial u_{2}}{\partial y}+\frac{\partial v_{2}}{\partial x}\right)+\epsilon_{2} E_{x}\left(-\frac{\partial \psi}{\partial y}\right) \\
p_{1}-p_{2}+2\left(\mu_{2} \frac{\partial v_{2}}{\partial y}-\mu_{1} \frac{\partial v_{1}}{\partial y}\right)+\frac{\epsilon_{2}}{2}\left[\left(-\frac{\partial \psi}{\partial y}\right)^{2}-E_{x}^{2}\left(1-\frac{\epsilon_{1}}{\epsilon_{2}}\right)\right]=0,
\end{gathered}
$$


where $\epsilon_{1}$ and $\epsilon_{2}$ are the permittivities of fluids "1" and "2", respectively. To obtain the normalized form, the following dimensionless quantities (distinguished by an overhead caret) are introduced:

$$
(\hat{x}, \varepsilon \hat{y})=(x, y) / L, \quad(\hat{u}, \hat{v})=(u, v) / u_{0}, \quad \hat{p}=\varepsilon^{2} p L / \mu_{2} u_{0}, \quad \hat{\psi}=\psi / \zeta_{0},
$$

in which $\varepsilon=h_{w} / L$ and $u_{0}=-\epsilon_{2} E_{x} \zeta_{0} / \mu_{2}$. The dimensionless form of Eqs. (49) and (50) can be expressed as

$$
\begin{gathered}
\frac{\partial \hat{u}_{1}}{\partial \hat{y}}+\varepsilon^{2} \frac{\partial \hat{v}_{1}}{\partial \hat{x}}=\eta\left(\frac{\partial \hat{u}_{2}}{\partial \hat{y}}+\varepsilon^{2} \frac{\partial \hat{v}_{2}}{\partial \hat{x}}\right)+\eta \frac{\partial \hat{\psi}}{\partial \hat{y}} \\
\hat{p}_{1}-\hat{p}_{2}+2 \varepsilon^{2}\left(\frac{\partial \hat{v}_{2}}{\partial \hat{y}}-\frac{1}{\eta} \frac{\partial \hat{v}_{1}}{\partial \hat{y}}\right)-\frac{\varepsilon}{2}\left[\frac{1}{\beta}\left(-\frac{\partial \hat{\psi}}{\partial \hat{y}}\right)^{2}-\beta\left(1-\frac{\epsilon_{1}}{\epsilon_{2}}\right)\right]=0,
\end{gathered}
$$

in which the dimensionless parameter $\beta=\varepsilon E_{x} L / \zeta_{0}$ is a ratio of the Maxwell stress to the viscous stress, where the two stresses are assumed to be comparable to each other. As $\varepsilon \ll 1$ is a small parameter, Eqs. (52) and (53) reduce to

$$
\begin{gathered}
\frac{\partial \hat{u}_{1}}{\partial \hat{y}}=\eta \frac{\partial \hat{u}_{2}}{\partial \hat{y}}+\eta \frac{\partial \hat{\psi}}{\partial \hat{y}} \\
\hat{p}_{1}=\hat{p}_{2}
\end{gathered}
$$




\section{References}

[1] D. Ross, T. J. Johnson, L. E. Locascio, Imaging of electroosmotic flow in plastic microchannels, Anal. Chem. 73(11) (2001) 2509-2515.

[2] W. B. Zimmerman, J. M. Rees, T. Craven, Rheometry of non-Newtonian electrokinetic flow in a microchannel T-junction, Microfluid. Nanofluid. 2(6) (2006) 481-492.

[3] M. J. Pikal, The role of electroosmotic flow in transdermal iontophoresis, Adv. Drug Deliv. Rev. 46(1) (2001) 281-305.

[4] H. A. Stone, A. D. Stroock, A. Ajdari, Engineering flows in small devices, Annu. Rev. Fluid Mech. 36(1) (2004) 381-411.

[5] Y. Gao, T. N. Wong, J. C. Chai, C. Yang, K. T. Ooi, Numerical simulation of two-fluid electroosmotic flow in microchannels, Int. J. Heat Mass Tran. 284 (2005) 306-314.

[6] G. C. Shit, A. Mondal, A. Sinha, P. K. Kundu, Two-layer electro-osmotic flow and heat transfer in a hydrophobic micro-channel with fluid-solid interfacial slip and zeta potential difference, Colloids Surf. A: Physicochem. Eng. Asp. 506 (2016) 535-549.

[7] G. D. Ngoma, F. Erchiqui, Pressure gradient and electroosmotic effects on two immiscible fluids in a microchannel between two parallel plates, J. Micromech. Microeng. 16 (2006) 83-91.

[8] A. Brask, G. Goranovic, H. Bruus, Electroosmotically driven two-liquid viscous pump for no conducting liquids, Tech. Proc. Nanotech. 1 (2003) 190-193.

[9] M. Watanabe, H. Shirai, T. Hirai, Liquid-liquid two-layer electrohydrodynamic flow system, Sens. Actuator B-Chem. 94 (2003) 267-270.

[10] V. Shankar, A. Sharma, Instability of the interface between thin fluid films subjected to electric fields, J. Colloid Interface Sci. 274 (2004) 294-308.

[11] R. Verma, A. Sharma, K. Kargupta, J. Bhaumik, Electric field induced instability and pattern formation in thin liquid films, Langmuir 21 (2005) 3710-3721. 
[12] Y. Gao, T. N. Wong, C. Yang, K. T. Ooi, Transient two-liquid electroosmotic flow with electric charges at the interface Colloids Surf. A: Physicochem. Eng. Asp. 266 (2005) $117-128$.

[13] H. Li, T. N. Wong, N. T. Nguyen, Analytical model of mixed electroosmotic/pressure driven three immiscible fluids in a rectangular microchannel, Int. J. Heat Mass Tran. 52 (2009) 4459-4469.

[14] H. Li, T. N. Wong, N. T. Nguyen, Time-dependent model of mixed electroosmotic/pressure-driven three immiscible fluids in a rectangular microchannel, Int. J. Heat Mass Tran. 53 (2010) 772-785.

[15] M. Liu, Y. Liu, Q. Guo, J. Yang, Modeling of electroosmotic pumping of nonconducting liquids and biofluids by a two-phase flow method, J. Electroanal. Chem. 636 (2009) $86-92$.

[16] W. S. Choi, A. Sharma, S. Qian, G. Lim, S. W. Joo, On steady two-fluid electroosmotic flow with full interfacial electrostatics, J. Colloid Interface Sci. 357 (2011) 521-526.

[17] H. Gaikwad, D. N. Basu, P. K. Mondal, Electroosmotic transport of immiscible binary system with a layer of non-conducting fluid under interfacial slip: The role applied pressure gradient, Electrophoresis 37 (2016) 1998-2009.

[18] S. Mandal, U. Ghosh, A. Bandopadhyay, S. Chakraborty, Electro-osmosis of superimposed fluids in the presence of modulated charged surfaces in narrow confinements, J. Fluid Mech. 776 (2015) 390-429.

[19] A. Ajdari, Electro-osmosis on inhomogeneously charged surfaces, Phys. Rev. Lett. 75 (1995) 755-758.

[20] A. Ajdari, Generation of transverse fluid currents and forces by an electric field: electroosmosis on charge-modulated and undulated surfaces, Phys. Rev. E 53 (1996) 49965005.

[21] C. Chen, C. Cho, Electrokinetically-driven flow mixing in microchannels with wavy surface, J. Colloid Interface Sci. 312 (2007) 470-480. 
[22] X. Xuan, D. Li, Electroosmotic flow in microchannels with arbitrary geometry and arbitrary distribution of wall charge, J. Colloid Interface Sci. 289(1) (2005) 291-303.

[23] S. Bhattacharyya, A. K. Nayak, Combined effect of surface roughness and heterogeneity of wall potential on electroosmosis in microfluidic/nanofluidic channels, J. Fluid. Eng. 132 (2010) 041103.

[24] C. O. Ng, C. Qi, Electroosmotic flow of a power-law fluid in a non-uniform microchannel, J. Non-Newtonian Fluid Mech. 208 (2014) 118-125.

[25] S. Bhattacharyya, S. Bera, Combined electroosmosis-pressure driven flow and mixing in a microchannel with surface heterogeneity, Appl. Math. Model. 39(15) (2015) 43374350.

[26] H. Yoshida, T. Kinjo, H. Washizu, Analysis of electro-osmotic flow in a microchannel with undulated surfaces, Comput. Fluids 124 (2016) 237.

[27] W. M. Deen, Analysis of Transport Phenomena, second ed. Oxford University Press, New York, 2012.

[28] L. G. Leal, Advanced Transport Phenomena, Cambridge University Press, Cambridge, 2007.

[29] D. Long, H. A. Stone, A. Ajdari, Electroosmotic flows created by surface defects in capillary electrophoresis, J. Colloid Interface Sci. 212 (1999) 338-349.

[30] A. Ajdari, Transverse electrokinetic and microfluidic effects in micropatterned channels: Lubrication analysis for slab geometries, Phys. Rev. E 65 (2001) 016301.

[31] S. Ghosal, Lubrication theory for electro-osmotic flow in microfluidic channel of slowly varying cross-section and wall charge, J. Fluid Mech. 459 (2002) 103-128.

[32] D. Erickson, D. Li, C. Werner, An improved method of determining the $\zeta$-potential and surface conductance, J. Colloid Interface Sci. 232 (2000) 186-197.

[33] S. L. Barker, D. Ross, M. J. Tarlov, M. Gaitan, L. E. Locascio, Control of flow direction in microfluidic devices with polyelectrolyte multilayers, Anal. Chem. 72 (2000) 59255929. 
[34] A. D. Stroock, M. Weck, D. T. Chiu, W. T. Huck, P. J. Kenis, R. F. Ismagilov, G. M. Whitesides, Patterning electro-osmotic flow with patterned surface charge, Phys. Rev. Lett. 84 (2001) 3314.

[35] W. L. Hau, D. W. Trau, N. J. Sucher, M. Wong, Y. Zohar, Surface-chemistry technology for microfluidics, J. Mircomech. Microeng. 13 (2003) 272-278.

[36] E. Biddiss, D. Erickson, D. Li, Heterogeneous surface charge enhanced micromixing for electrokinetic flows, Anal. Chem. 76 (2004) 3208-3213.

[37] D. C. Duffy, J. C. McDonald, O. J. Schueller, G. M. Whitesides, Rapid prototyping of microfluidic systems in poly (dimethylsiloxane), Anal. Chem. 70 (1998) 4974-4984.

[38] Y. Gu, D. Li, An electrical suspension method for measuring the electric charge on small silicone oil droplets dispersed in aqueous solutions, J. Colloid Interface Sci. 195 (1997) 343-352.

[39] A. M. Afonso, M. A. Alves, F. T. Pinho, Analytical solution of two-fluid electro-osmotic flows of viscoelastic fluids, J. Colloid Interface Sci. 395 (2013) 277-286. 